\title{
A Novel Grey Power-markov Model for the Prediction of China's Electricity Consumption
}

\section{Liqin Sun ( $\square$ liqinsun@stu.xidian.edu.cn )}

Xidian University School of Mathematics and Statistics

\section{Youlong Yang}

Xidian University School of Mathematics and Statistics

\section{Tong Ning}

Xidian University School of Mathematics and Statistics

Jiadi Zhu

Xidian University School of Mathematics and Statistics

\section{Research Article}

Keywords: Grey power model, the Rolling mechanism, the Markov Theory, Electricity Consumption, Consumption prediction

Posted Date: July 23rd, 2021

DOI: https://doi.org/10.21203/rs.3.rs-648522/v1

License: (1) (1) This work is licensed under a Creative Commons Attribution 4.0 International License. Read Full License

Version of Record: A version of this preprint was published at Environmental Science and Pollution Research on November 12th, 2021. See the published version at https://doi.org/10.1007/s11356-02117016-1. 
5 Abstract

The grey prediction models of time series are widely used in demand forecasting because only limited data can be used to build the models and no statistical hypothesis is needed. In this paper, a grey power Markov prediction model $(\operatorname{RGPMM}(\lambda, 1,1))$ with time-varying parameters is proposed. This model is based on the principle of "new information priority", combined with rolling mechanism and Markov theory, and the prediction residual error is modified to further improve the prediction accuracy. Compared with the classic grey models, the new model not only overcomes the inherent defect of poor adaptability to the original data, but also uses real-time information to better reflect the nonlinear characteristics of the original data, so it can be used to describe and predict the nonlinear development trend of things. In order to verify the validity and applicability of the model, the proposed model is used to forecast the total electric consumption in China. The experimental results show that the proposed model has a better prediction effect than other grey models. The proposed model is used to forecast China's total electricity consumption in the next six years from 2018 to 2023. 
Ghani IMM et al. (Ghani and Ahmad, 2010) used SPSS software to establish a linear regression model based on the multiple regression method to predict and analyze the fish landing to demonstrate the effectiveness and feasibility of the method. Wang $\mathrm{J}$ et al. (Wang et al., 2018) used an autoregressive moving average (ARMA) model based on the time series algorithm to predict short-term wind power. Zhang Y et al. (Zhang et al., 2019) combined BP and RBF neural network methods to predict and analyze wind speed to verify the effectiveness and accuracy of the combined prediction model. Wang S et al. (Wang et al., 2020) used the grey management degree and grey theory to establish a grey system model based on the basic data of urban heating and forecast its demand. Guo H et al. (Guo et al., 2013) proposed a new comprehensive adaptive grey model, $\operatorname{CAGM}(1, N)$, which can be applied to any actual forecasting issues and can obtain higher fitting and prediction accuracy compared with the traditional GM(1,N) model (Pai et al., 2008, Tien, 2008).

These methods make predictions based on raw data by maximizing the fitting accuracy, but do not take into account the complex diversity of things themselves. The single grey model has a large error in the process of forecasting, and it is difficult to achieve the expected accuracy. And the Markov model is suitable for the prediction of random problems, it can better describe the dynamic trend of randomly changing objects and can make up for the shortcomings of the grey model. Therefore, two prediction models are combined. The grey model is used to forecast, and the predicted values are corrected by the Markov chain to effectively improve the accuracy of the prediction, so as to achieve the purpose of scientific prediction and analysis. So far, the combination of grey theory and Markov model has applied many areas of prediction, such as He Yong et al. (HE and BAO, 1992) put forward the grey Markov model for the first time by combining the advantages of grey model and Markov theory, which has since been widely used in the prediction of traffic, natural disasters, energy consumption and other fields. Kumar et al. (Kumar and Jain, 2019) combined the grey Markov and time series model to ground breakingly predict energy consumption in India, which provided a feasible scheme for the prediction of India's energy consumption. Cao Jian et al. (CAO et al., 2019) explored the internal relationship among the accidents of road transport on hazardous chemical and the traffic accidents in China based on Grey Markov model, and analyzed the Grey Markov combined prediction model in the prediction of safety accident. The effectiveness and feasibility of the method had been verified by experiments.

The above research shows that the grey Markov model has better prediction accuracy and ability. However, the actual operation found that when faced with the prediction of small sample data, the model still has a certain degree of contingency. Therefore, according to the characteristics of China's electricity consumption data, this paper optimizes and improves the traditional grey Markov model, and proposes $\operatorname{RGPMM}(\lambda, 1,1)$ to predict China's electricity consumption more accurately, so as to provide more accurate information for the rational distribution of energy.

\section{1. objective}

This article has carried out the work in the following aspects:

1)Propose a grey power model (Wang et al., 2011) with non-integer powers $\lambda$. In the traditional grey power model, $\lambda$ is generally taken as an integer. While in this thesis, $\lambda$ belongs to the real number $\mathrm{R}$, that is, $\lambda$ can be taken the fraction to establish the model. The value of $\lambda$ can be estimated through the optimization theory, and then a non-integer 
grey power prediction model can be established.

2)The introduction of Rolling Mechanism (Akay and Atak, 2007). In the forecasting process, because the data from the far past has little effect on the forecast, the Rolling Mechanism is introduced to continuously update the input information, which breaks the constraint of constant initial value in the classic grey prediction model and complies with the principle of "new information priority" (D, 1989).

3)In this paper, the relative error of the grey power model is used as the index, and the weighted Markov theory (Liu et al., 2018) is used to correct the grey power model, which further improves the prediction accuracy and adaptability of the model.

\subsection{Organization}

The rest of this article is organized as follows: Section 2 briefly introduces the historical background of the grey model and the traditional GM(1,1) model, Markov theory, Rolling Mechanism and the grey development zone. Section 3 introduces how to build the $\operatorname{RGPMM}(\lambda, 1,1)$. Section 4 illustrates the practicability of the $\operatorname{RGPMM}(\lambda, 1,1)$ by experiment, and forecasts the total electricity consumption in the next few years by this model. Section 5 contains the conclusions and suggestions for future work.

\section{Basic knowledge}

\subsection{Basic $\operatorname{GM}(1,1)$}

In 1982, Professor Deng Julong first proposed the concept of grey system and built the $\operatorname{GM}(1,1)$. The process of the $\operatorname{GM}(1,1)$ is as follows (D, 1982, Lin et al., 2012, Zeng et al., 2020):

Step 1: Transforing the original data. Let a set of non-negative sequences is $X^{(0)}=$ $\left\{x^{(0)}(1), x^{(0)}(2), \cdots, x^{(0)}(n)\right\},(n \geq 4)$. The 1-AGO sequence is given by

$$
X^{(1)}=\left\{x^{(1)}(1), x^{(1)}(2), \cdots, x^{(1)}(n)\right\}
$$

where, $x^{(1)}(k)=\sum_{i=1}^{k} x^{(0)}(i), k=1,2, \cdots, n$.

Step 2: Based on the sequence $X^{(1)}$, the whitening form equation of the prediction model can be established:

$$
\frac{d x^{(1)}}{d t}+a \cdot x^{(1)}=b .
$$

In Formula (2), $a$ and $b$ are the parameters to be estimated. The grey differential equation is:

$$
x^{(0)}(k)+a \cdot z^{(1)}(k)=b,
$$

where, $z^{(1)}(k)=\frac{1}{2} \cdot\left[x^{(1)}(k)+x^{(1)}(k-1)\right]$ is the background value and $Z^{(1)}=\left\{z^{(1)}(2), z^{(1)}(3), \cdots, z^{(1)}(n)\right\}$ is the mean sequence of $X^{(1)}$ (ping Xiong et al., 2014). 
Step 3: Estimating the model parameters. Set the parameters vector to be estimated as $\left(\begin{array}{l}\hat{a} \\ \hat{b}\end{array}\right)$ and solve it according to the least squares method to obtain

$$
\left(\begin{array}{l}
\hat{a} \\
\hat{b}
\end{array}\right)=\left(B^{T} B\right)^{-1} B^{T} Y
$$

${ }_{98} \quad$ where $B=\left(\begin{array}{cc}-z^{(1)}(2) & 1 \\ -z^{(1)}(3) & 1 \\ \cdots & \cdots \\ -z^{(1)}(n) & 1\end{array}\right), Y=\left(x^{(0)}(2), x^{(0)}(3), \cdots, x^{(0)}(n)\right)^{T}$.

Step 4: Obtaining the time response function. According to Equation (4), solve Equation (2), then the time corresponding equation is computed as:

$$
\hat{x}^{(1)}(t+1)=\left(x^{(0)}(1)-\frac{b}{a}\right) \cdot e^{-a t}+\frac{b}{a}, t=1,2, \cdots, n, n+1, \cdots .
$$

Step 5: Obtaining the fitted and predicted values in the original domain. The simplified predicted value of the first-order accumulation operator sequence is $\hat{x}^{(0)}(t)=\hat{x}^{(1)}(t)-$ $\hat{x}^{(0)}(t-1)$, namely,

$$
\hat{x}^{(0)}(t)=\left[x^{(0)}(1)-\frac{\hat{b}}{\hat{a}}\right] \cdot\left(1-e^{\hat{a}}\right) \cdot e^{-\hat{a}(t-1)}, t=2,3, \cdots, n, n+1, \cdots .
$$

where $\hat{x}^{(0)}(t)(t \leq n)$ are called fitted values, and $\hat{x}^{(0)}(t)(t>n)$ are called predicted values.

\subsection{Markov process}

Markov process (Zhao et al., 2014) is a theory that studies the state of things and their transition. A Markov process in which time and state are both discrete is called a Markov chain. Markov chain analysis is a statistical analysis method based on the probability theory and stochastic process theory, using stochastic mathematical models to analyze the quantitative relationship of objective objects in the development and change process. Its characteristic is no after-effect, that is, the current state of the system is only related to the previous state, and has nothing to do with the subsequent state.

\subsubsection{Transition probability and Transition probability matrix}

In Markov process, the transition probability and the transition probability matrix of states need to be calculated, which are defined as follows:

Definition 1. Let $\left\{X_{n}, n \in T\right\}$ be a Markov chain, and call the conditional probability $p_{i j}(n, T)=P\left(X_{n+1} \mid X_{n}=i\right), i, j \in T$ the one-step transition probability of the Markov chain $\left\{X_{n}, n \in T\right\}$ at time $n$, which is referred to as the transition probability. That is, the conditional probability that the particle is in state $i$ at time $n$ and then is in state $j$ after one step. The matrix composed of transition probability is the transition probability matrix. In 
a Markov chain, the system state transition can be represented by the transition probability matrix $P$ as follows:

$$
P=\left(\begin{array}{cccc}
p_{11} & p_{12} & \cdots & p_{1 n} \\
p_{21} & p_{22} & \cdots & p_{2 n} \\
\vdots & \vdots & \ddots & \vdots \\
p_{n 1} & p_{n 2} & \cdots & p_{n n}
\end{array}\right)
$$

Definition 2. (The Grey Power Model) Assuming $X^{(0)}$ is a non-negative unimodal raw data sequence, $X^{(1)}$ is the $1-A G O$ sequence of $X^{(0)} . Z^{(1)}$ is a sequence generated next to the mean of the $X^{(1)}$. Then, there is the following non-linear model which meets the three conditions of gray modeling, and the grey power model is

$$
x^{(0)}(k)+a \cdot z^{(1)}(k)=b \cdot\left[z^{(1)}(k)\right]^{\lambda}
$$

The whitening equation of the grey power model is

$$
\frac{d x^{(1)}}{d t}+a \cdot x^{(1)}=b \cdot\left[x^{(1)}\right]^{\lambda}
$$

Solving the above model by the solution method of $\operatorname{GM}(1,1)$, we can get the solution of the whitening equation is

$$
x^{(t+1)}=\left\{e^{-(1-\lambda) a t}\left[(1-\lambda) \int b e^{(1-\lambda) a t} d t+c\right]\right\}^{\frac{1}{1-\lambda}} .
$$


146 147

\subsection{Parameters analysis of $\operatorname{GPM}(\lambda, 1,1)$}

\subsubsection{Parameter $\lambda$ estimation method}

The parameter $\lambda$ is an important coefficient in the $\operatorname{GPM}(\lambda, 1,1)$. According to the above formulas, since $x^{(1)} \neq 0$, divide both sides of Equation (5) by $\left[x^{(1)}\right]^{\lambda}$ and then take the deriation about $t$ to get Equation (7) as follows:

$$
\frac{d^{2} x^{(1)}}{d t^{2}} \cdot\left[x^{(1)}\right]^{\lambda}-\lambda \cdot\left(\frac{d x^{(1)}}{d t}\right)^{2}\left[x^{(1)}\right]^{\lambda-1}=-a(1-\lambda) \cdot\left[x^{(1)}\right]^{\lambda} \cdot \frac{d x^{(1)}}{d t}
$$

According to the information coverage principle of grey derivative, we cover $\frac{d x^{(1)}}{d t}$ and $\frac{d^{2} x^{(1)}}{d t^{2}}$ in Equation (7) with the first grey derivatives and the second grey derivatives of $x^{(1)}$, then we will get

$$
\begin{aligned}
& {\left[x^{(0)}(t)-x^{(0)}(t-1)\right] \cdot\left[z^{(1)}(t)\right]^{\lambda}-\lambda \cdot\left[x^{(0)}(t)\right]^{2} \cdot\left[z^{(1)}(t)\right]^{\lambda-1}} \\
& =-a(1-\lambda) \cdot\left[z^{(1)}(t)\right]^{\lambda} \cdot x^{(0)}(t)
\end{aligned}
$$

Dividing the Equation (8) with $t=k$ by the Equation (8) with $t=k+1$, we can eliminate the unknown parameter $a$ and get

$$
\begin{aligned}
& \frac{\left[x^{(0)}(k)-x^{(0)}(k-1)\right] \cdot\left[z^{(1)}(k)\right]^{\lambda}-\lambda \cdot\left[x^{(0)}(k)\right]^{2} \cdot\left[z^{(1)}(k)\right]^{\lambda-1}}{\left[x^{(0)}(k+1)-x^{(0)}(k)\right] \cdot\left[z^{(1)}(k+1)\right]^{\lambda}-\lambda \cdot\left[x^{(0)}(k+1)\right]^{2} \cdot\left[z^{(1)}(k+1)\right]^{\lambda-1}} \\
& =\frac{\left[z^{(1)}(k)\right]^{\lambda} \cdot x^{(0)}(k)}{\left[z^{(1)}(k+1)\right]^{\lambda} \cdot x^{(0)}(k+1)}
\end{aligned}
$$

It follows from Equation (9) that

$$
\begin{aligned}
\lambda= & \frac{\left[x^{(0)}(k+1)-x^{(0)}(k)\right] \cdot z^{(1)}(k+1) \cdot z^{(1)}(k) \cdot x^{(0)}(k)}{\left[x^{(0)}(k+1)\right]^{2} \cdot z^{(1)}(k) \cdot x^{(0)}(k)-\left[x^{(0)}(k)\right]^{2} \cdot z^{(1)}(k+1) \cdot x^{(0)}(k+1)} \\
& -\frac{\left[x^{(0)}(k)-x^{(0)}(k-1)\right] \cdot z^{(1)}(k) \cdot z^{(1)}(k+1) \cdot x^{(0)}(k+1)}{\left[x^{(0)}(k+1)\right]^{2} \cdot z^{(1)}(k) \cdot x^{(0)}(k)-\left[x^{(0)}(k)\right]^{2} \cdot z^{(1)}(k+1) \cdot x^{(0)}(k+1)}
\end{aligned}
$$

From the expression of $\lambda$, we can see that it can not only reflect the grey derivative of the original data, but also reflect the role of grey integral. When $k=2,3, \cdots, n-1$, the corresponding $(n-2)$ values of $\lambda$ can be computed, which is $\left\{\lambda_{k}\right\}$.

Let $g(\lambda)=\sum_{k=2}^{n-1}\left(\lambda-\lambda_{k}\right)^{2}$, the value of $\lambda$ that makes $g(\lambda)$ take the minimum value is the constant value to be determined.

Since $g(\lambda)$ is a parabola with an opening upward, according to the first order condition of unconditional extremum, the optimal value of $\lambda$ is

$$
\hat{\lambda}=\frac{1}{n-2} \sum_{k=2}^{n-1} \lambda_{k}
$$

In this case, $g(\hat{\lambda})$ takes the minimum value. 
153

\subsubsection{Estimates of parameters $a$ and $b$}

After the optimal value of $\lambda$ is determined, the parameters $a$ and $b$ can be estimated directly according to the least square method. Then, we can get the theorem 1 .

Theorem 1. Assuming $X^{(0)}, X^{(1)}$ and $Z^{(1)}$ are as defined in Definition 1, then the least squares estimate of the parameter sequence in $\operatorname{GPM}(\lambda, 1,1)$ is

$$
\left(\begin{array}{l}
\hat{a} \\
\hat{b}
\end{array}\right)=\left(B^{T} B\right)^{-1} B^{T} Y,
$$

where $B=\left(\begin{array}{cc}-z^{(1)}(2) & {\left[z^{(1)}(2)\right]^{\hat{\lambda}}} \\ -z^{(1)}(3) & {\left[z^{(1)}(3)\right]^{\hat{\lambda}}} \\ \cdots & \cdots \\ -z^{(1)}(n) & {\left[z^{(1)}(n)\right]^{\hat{\lambda}}}\end{array}\right), Y=\left(x^{(0)}(2), x^{(0)}(3), \cdots, x^{(0)}(n)\right)^{T}$.

\subsection{Solution of $\operatorname{GPM}(\lambda, 1,1)$}

According to Equation (12) and the estimation results of parameters, we can simplify it to get $\hat{x}^{(1)}(t+1)=\left[c \cdot e^{-(1-\hat{\lambda}) \hat{a} t}+\hat{b} / \hat{a}\right]^{\frac{1}{1-\hat{\lambda}}}$. If the initial value $\hat{x}^{(1)}(1)=x^{(0)}(1)$, then the solution of $\operatorname{GPM}(\lambda, 1,1)$ is

$$
\hat{x}^{(1)}(t+1)=\left\{\left[\left(x^{(0)}(1)\right)^{1-\hat{\lambda}}-\hat{b} / \hat{a}\right] \cdot e^{-(1-\hat{\lambda}) \hat{a} t}+\hat{b} / \hat{a}\right\}^{\frac{1}{1-\lambda}} .
$$

\subsection{Rolling modeling mechanism}

It is easy to produce some unacceptable errors in practical applications. In order to reduce the errors, Rolling Mechanism is proposed. The length of training data set is set as $c$, and the predicted period of rolling modeling is set as $d$. The steps are as follows:

Step 1:The original sequence $\left\{x^{(0)}(1), x^{(0)}(2), \cdots, x^{(0)}(c)\right\}$ is used to model and the $d$-period prediction value $\left\{\hat{x}^{(0)}(c+1), \hat{x}^{(0)}(c+2), \cdots, \hat{x}^{(0)}(c+d)\right\}$ is obtained;

Step 2: When predicting the sequence $\left\{\hat{x}^{(0)}(c+d+1), \hat{x}^{(0)}(c+d+2), \cdots, \hat{x}^{(0)}(c+2 d)\right\}$, we use the latest $c$ data points $\left\{\hat{x}^{(0)}(d+1), \hat{x}^{(0)}(d+2), \cdots, \hat{x}^{(0)}(d+c)\right\}$ to predict;

Step 3:Repeat step 2 and use the latest sequence to predict the next set of $d$ data points until the required data points are predicted.

The flow chart is shown in Fig.1. 


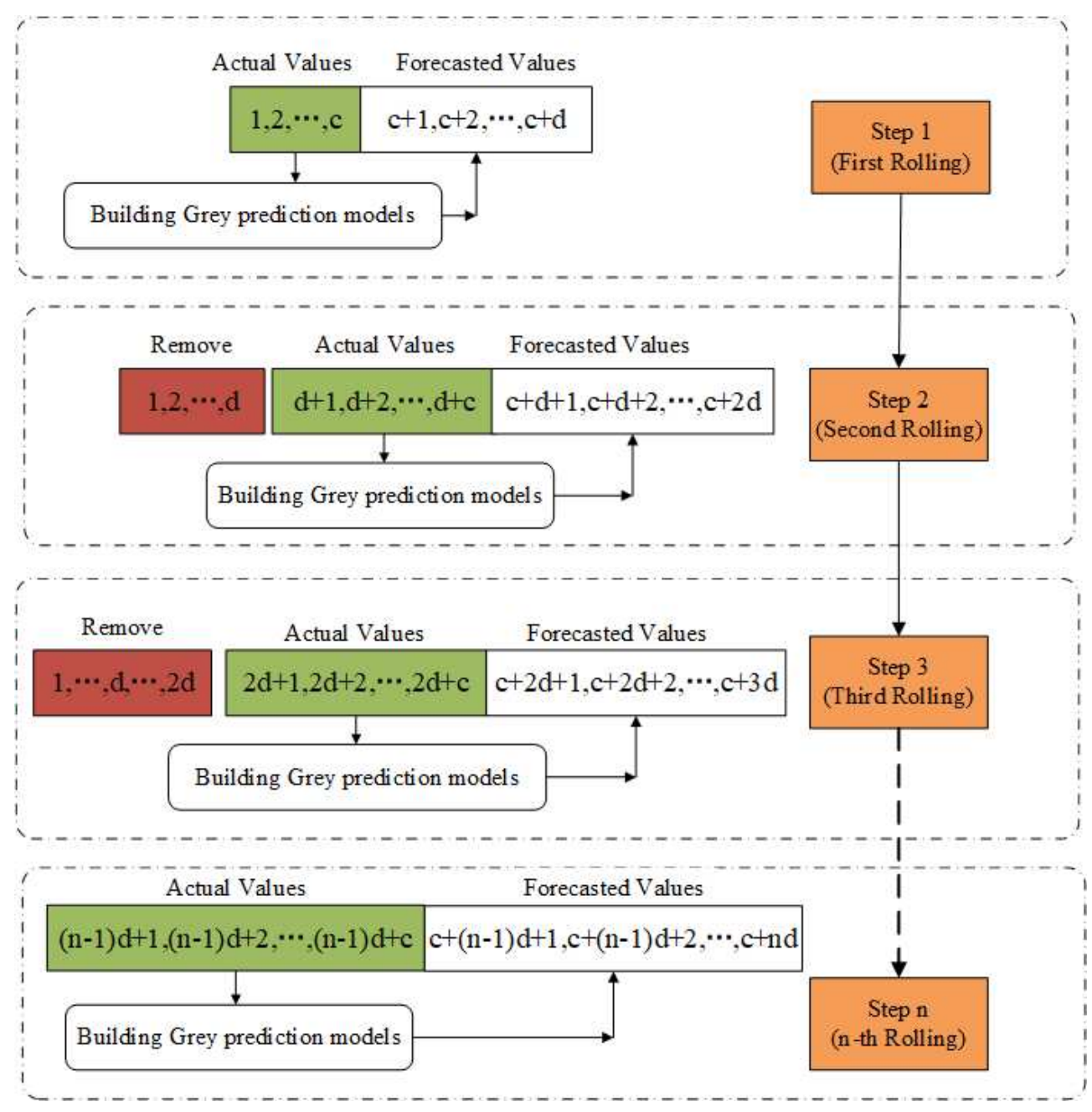

Fig. 1. The Rolling Mechanism

\subsection{Building the $\operatorname{RGPMM(\lambda ,1,1)}$}

Due to the complexity of the real situation, there will always be a certain difference between the fitting value obtained by $\operatorname{GPM}(\lambda, 1,1)$ and the real value. Then, the accuracy index of grey fitting is random and non-stationary. In order to correct the predictable result and improve the prediction accuracy of $\operatorname{GPM}(\lambda, 1,1)$, the fluctuation of grey fitting accuracy index is analyzed and predicted by Markov theory in this paper. Combined with the rolling mechanism, the electricity consumption is forecasted in the future. The steps of $\operatorname{RGPMM}(\lambda, 1,1)$ are as follows and the flow chart is shown in Fig.2.

Step 1:Calculate fitted values and predicted values. According to the time series $X^{(0)}=\left\{x^{(0)}(1), x^{(0)}(2), \cdots, x^{(0)}(n)\right\}, \operatorname{GPM}(1,1)$ is established to obtain

$$
\begin{aligned}
\hat{x}^{(0)}(t+1)= & \left\{\left[\left(x^{(0)}(1)\right)^{1-\hat{\lambda}}-\frac{\hat{b}}{\hat{a}}\right] \cdot e^{-(1-\hat{\lambda}) a t}+\frac{\hat{b}}{\hat{a}}\right\}^{\frac{1}{1-\hat{\lambda}}} \\
& -\left\{\left[\left(x^{(0)}(1)\right)^{1-\hat{\lambda}}-\frac{\hat{b}}{\hat{a}}\right] \cdot e^{-(1-\hat{\lambda}) a(t-1)}+\frac{\hat{b}}{\hat{a}}\right\}^{\frac{1}{1-\hat{\lambda}}}
\end{aligned}
$$


where $\hat{x}^{(0)}(t)(t \leq n)$ are called fitted values, and $\hat{x}^{(0)}(t)(t>n)$ are called predicted values.

Step 2:Calculate the grey fitting accuracy index. The grey fitting accuracy index is set to $Y(t)=x^{(0)}(t) / \hat{x}^{(0)}(t)$, which reflects the deviation degree of the data fitted by model from the original data.

Step 3: Division of state interval. Considering that $Y(t)$ is divided into $m$ state $E_{i}=\left[\otimes_{1 i}, \otimes_{2 i}\right], i=1,2, \cdots, m$. The grey elements $\otimes_{1 i}$ and $\otimes_{2 i}$ are the lower bound and the upper bound of the $i$-th state, where $\otimes_{1 i}=Y(t)+a_{i} \bar{Y}, \otimes_{2 i}=Y(t)+b_{i} \times \bar{Y}, \bar{Y}=\frac{1}{n} \cdot \sum_{i=1}^{n} Y(i)$. The $a_{i}$ and $b_{i}$ are constants that need to be determined based on experience and data.

Considering the limited amount of electricity consumption data in this paper, it is more appropriate to use the cluster analysis to determine the number of classification classes and the classification interval.

Step 4: Establish a state transition matrix. The transition probability of state $E_{i}$ to state $E_{j}$ is

$$
p_{i j}(\omega)=\frac{M_{i j}(\omega)}{M_{i}} .
$$

where, $M_{i j}(\omega)$ is the number of samples $Y(t)$ transferred from the state of $E_{i}$ to the state of $E_{j}$ through $\omega$ steps; $M_{i}$ is the total number of occurrences of $E_{i}$, and satisfies $\sum_{j=1}^{m} p_{i j}(\omega)=1$, $i, j=1,2, \cdots, m$. Therefore, $p_{i j}(\omega)$ reflects the probability of transition from $E_{i}$ to $E_{j}$ through $\omega$ steps.

The state transition probability matrix is

$$
R(\omega)=\left(\begin{array}{cccc}
p_{11}(\omega) & p_{12}(\omega) & \cdots & p_{1 m}(\omega) \\
p_{21}(\omega) & p_{22}(\omega) & \cdots & p_{2 m}(\omega) \\
\vdots & \vdots & \ddots & \vdots \\
p_{m 1}(\omega) & p_{m 2}(\omega) & \cdots & p_{m m}(\omega)
\end{array}\right)
$$

The $R(\omega)$ reflects the transfer law between the various states of the system. By examining $R(\omega)$ and the current state, we can predict the future development and change of the system.

The autocorrelation coefficient of each order is

$$
r_{\omega}=\frac{\sum_{l=1}^{n-\omega}[Y(l)-\bar{Y}] \cdot[Y(l+\omega)-\bar{Y}]}{\sum_{l=1}^{n}[Y(l)-\bar{Y}]^{2}}
$$

By normalizing $r_{\omega}$, the Markov weight of each order is

$$
\theta_{\omega}=\frac{\left|r_{\omega}\right|}{\sum_{\omega=1}^{m}\left|r_{\omega}\right|}, \omega \leq m .
$$

where, $\theta_{\omega}$ is the Markov weight of the $\omega$ th order, and the $\omega$ th order generally is the maximum order when $\left|r_{\omega}\right| \geq 0.3$.

Step 5: Calculate more accurate the predicted value. The transition probability matrix is used to predict the state interval $E_{i}$ of the grey fitting precision index. The interval 
interpolation is used to determine the predicted value. Therefore, $\tilde{x}^{(0)}(n+1)=\hat{x}^{(0)}(n+1) *$ $\widehat{Y}(n+1)$, where

$$
\widehat{Y}(n+1)=\otimes_{1 i} \times \frac{p_{i-1}}{p_{i-1} * p_{i+1}}+\otimes_{2 i} \times \frac{p_{i+1}}{p_{i-1} * p_{i+1}}
$$

Step 6:Calculate more accurate predicted values in the next few years. Through a rolling mechanism, the input data is updated and the $\operatorname{RGMM}(\lambda, 1,1)$ is established to forecast the next year's value. Continue the above process until the desired data forecasted.

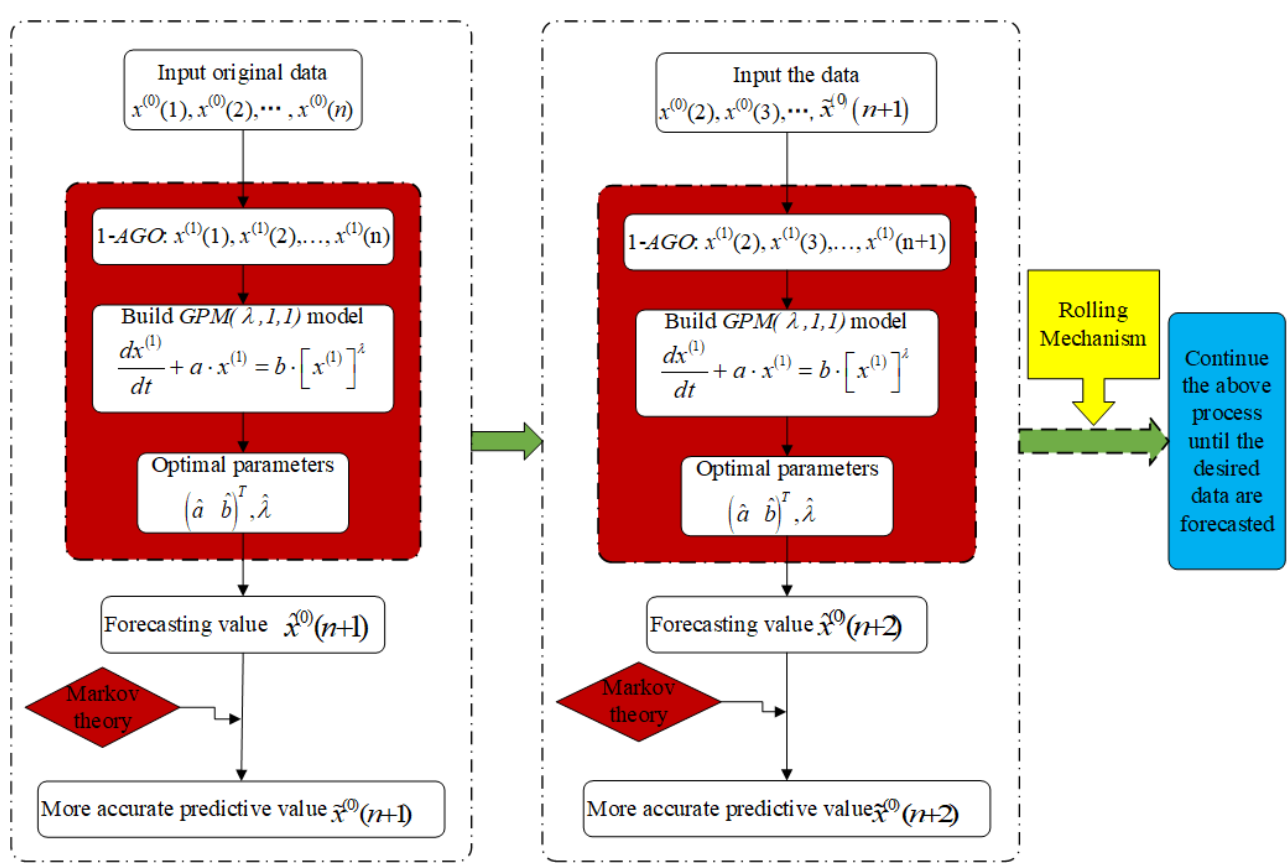

Fig. 2. The flow chart of $\operatorname{RGPMM}(\lambda, 1,1)$

\subsection{Evaluation Metrics}

Here are three kinds of evaluation metrics to evaluate the prediction accuracy. Only when the three metrics are all passed, the $\operatorname{RGPMM}(\lambda, 1,1)$ can be used to predict, and its predicted values have reference significance. There are three kinds of evaluation metrics as follows:

\section{A: The residual test.}

The three statistical indicators are determined, namely MAE(Mean Absolute Error) (Hamzaçebi, 2007), MAPE(Mean Absolute Percentage Error) (Azadeh et al., 2008), and RMSE(The Root Mean Squared Error) (Geem and Roper, 2009). The formulas for MAE,MAPE and RMSE are as follows:

$$
\begin{aligned}
& M A E=\frac{1}{n} \cdot \sum_{i=1}^{n}\left|x^{(0)}(i)-\hat{x}^{(0)}(i)\right|, M A P E=\frac{1}{n} \cdot \sum_{i=1}^{n}\left|\frac{x^{(0)}(i)-\hat{x}^{(0)}(i)}{x^{(0)}(i)}\right| \\
& R M S E=\sqrt{\frac{1}{n} \cdot \sum_{i=1}^{n}\left(x^{(0)}(i)-\hat{x}^{(0)}(i)\right)^{2}}
\end{aligned}
$$

where $x^{(0)}(i)$ is the original value at time $i$, and the $\hat{x}^{(0)}(i)$ is the fitted value at time $i$. Table 1 shows criteria of forecasting performance. 
Table 1. MAPE criteria for model evaluation

\begin{tabular}{l|l}
\hline MAPE(\%) & Forecasting ability \\
\hline$<10$ & Highly accurate forecasting \\
$10-20$ & Good forecasting \\
$20-50$ & Reasonable forecasring \\
$>50$ & Weak and inaccurate forecasting \\
\hline
\end{tabular}

Table 2. the gradation of prediction accuracy

\begin{tabular}{l|l|l}
\hline P-value & C-value & Gradation \\
\hline$>0.95$ & $<0.35$ & Good \\
$>0.85$ & $<0.50$ & Qualified \\
$>0.70$ & $<0.65$ & Barely qualified \\
$\leq 0.70$ & $\geq 0.65$ & Unqualified \\
\hline
\end{tabular}

\section{B: The correlation degree.}

The test method of correlation degree is $\gamma_{g}$ :

$$
\gamma_{g}=\frac{1}{n-1} \cdot \sum_{i=1}^{n} \gamma_{g}(i)=\frac{1}{n-1} \cdot \sum_{i=1}^{n} \frac{\min \{\Delta(i)\}+\rho \cdot \max \{\Delta(i)\}}{\Delta(i)+\rho \cdot \max \{\Delta(i)\}}
$$

where, $\rho=0.5, \Delta(i)=\left|\hat{x}^{(0)}(i)-x^{(0)}(i)\right|, i=1,2, \cdots, n, n$ is the number of samples.

\section{C: The posterior error test.}

The process of posterior error test is as follows:

Step 1: Calculate $S_{0}=\sqrt{\frac{\sum_{i=1}^{n}\left(X^{(0)}(i)-\bar{X}^{(0)}\right)^{2}}{n-1}}$ of $\left\{X^{(0)}(i)\right\}$ and $S_{1}=\sqrt{\frac{\sum_{i=1}^{n}\left(\varepsilon^{(0)}(i)-\bar{\varepsilon}^{(0)}\right)^{2}}{n-1}}$ of $\left\{\varepsilon^{(0)}(i)\right\}$, where $\bar{X}^{(0)}=\frac{1}{n} \cdot \sum_{i=1}^{n} X^{(0)}(i), \varepsilon^{(0)}(i)=x^{(0)}(i)-\hat{x}^{(0)}(i), \bar{\varepsilon}^{(0)}=\frac{1}{n} \cdot \sum_{i=1}^{n} \varepsilon^{(0)}(i)$, $i=1,2, \cdots, n$

Step 2: The standard deviation ratio $C=\frac{S_{1}}{S_{0}}$;

Step 3: The error probability $P=\left\{\left|\varepsilon^{(0)}(i)-\bar{\varepsilon}^{(0)}(i)\right|<0.6745 \times S_{0}\right\}$;

Step 4: The discrimination rules are shown in Table 2.

\section{Case studies on forecasting the total electricity consumption in China}

Since forecasting electricity consumption is important for the dispatch and operation of the power system, the total electricity consumption forecast at the national level is put forward. To prove the prediction accuracy of $\operatorname{RGPMM}(\lambda, 1,1)$ in Section 3, it is compared with $\operatorname{GM}(1,1), \operatorname{GM}\left(1,1, X_{n}\right)$ (Bahrami et al., 2014, G et al., 2004), OICGM(1,1) (Akdi et al., 2020), and NOGM(1,1) models (Ding et al., 2018).

\subsection{Experimental data}

The experimental data is from the China Statistical Yearbook published by the National statistics Bureau of China (Bureau, 2019). The experimental data set includes the total amount of electricity consumption from 2008 to 2017, as shown in Table 3.

Selecting the input data set and determining the length of the input data set are the prerequisites to affect the accuracy of the prediction model. In this paper, the optimized subset method proposed by Wang (Wang et al., 2011) is used to determine the optimal length of the input data set, and $c=8$ can be obtained. 8 data points are used as input data points, and the data of the last two years are used to test the prediction effect of the model.

Table 3. Total electricity energy consumption in China

\begin{tabular}{l|c|c|c|c|c}
\multicolumn{7}{c}{ from 2008 to $2017\left(10^{12} \mathrm{kwh}\right)$} \\
\hline Year & 2008 & 2009 & 2010 & 2011 & 2012 \\
\hline actual value & 3.45414 & 3.70322 & 4.19345 & 4.70009 & 4.97626 \\
\hline Year & 2013 & 2014 & 2015 & 2016 & 2017 \\
\hline actual value & 5.42034 & 5.63837 & 5.80200 & 6.12971 & 6.48210 \\
\hline
\end{tabular}


4.2. The prediction results of experiment

In Table 3, the original sequence is $X^{(0)}=(3.45414,3.70322,4.19345,4.70009,4.97626$, $5.42034,5.63837,5.80200,6.12971,6.48210)$. The flow chart of using the $\operatorname{RGPM}(\lambda, 1,1)$ model to predict China's electricity consumption is shown in Fig.3.

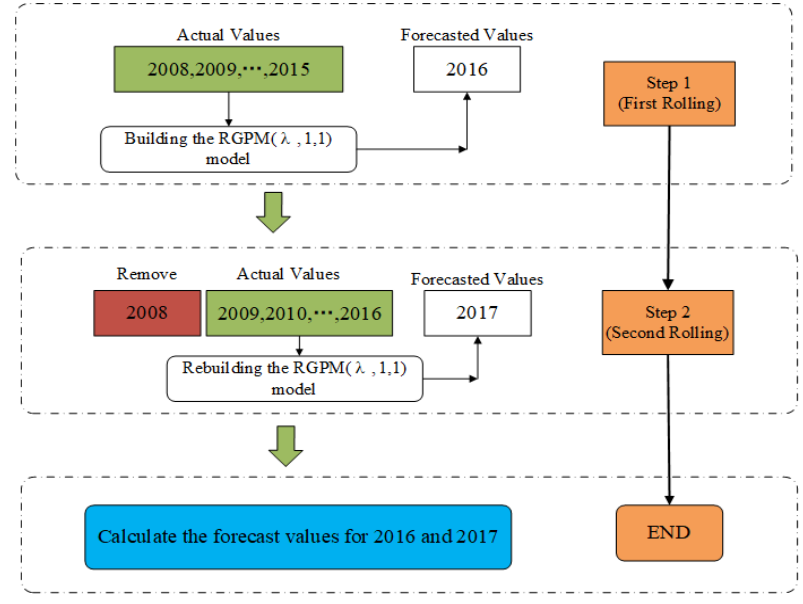

Fig. 3. The Rolling Mechanism

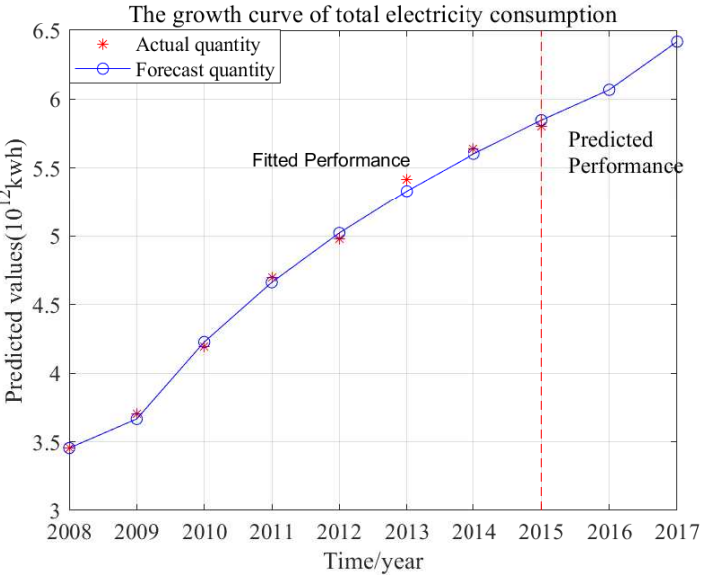

Fig. 4. The predicted value of $\operatorname{RGPM}(\lambda, 1,1)$

According to the Theorem 1 and the Formula (11) in section 3, the parameters of $\lambda$ and $\left(\begin{array}{l}a \\ b\end{array}\right)$ are estimated twice by MATLAB software programming to predict the total electricity consumption in 2016, 2017. Its calculation and results are as follows:

In 2016, the estimates calculated by MATLAB are $\hat{\lambda}=0.2539$ and $\left(\begin{array}{l}\hat{a} \\ \hat{b}\end{array}\right)=\left(\begin{array}{l}0.0041 \\ 2.4323\end{array}\right)$.

Substitute the parameter estimates into Equation (13), the time response sequence function by $\operatorname{RGPM}(\lambda, 1,1)$ model in 2016 is

$$
\hat{x}^{(1)}(t+1)=\left\{\left[\left(x^{(0)}(1)\right)^{(1-0.2539)}-\frac{2.4323}{0.0041}\right] * e^{-(1-0.2539) * 0.0041 * t}+\frac{2.4323}{0.0041}\right\}^{\frac{1}{(1-0.2539)}}
$$

Similarly, by entering the new initial value, the estimated values of parameters in 2017 are $\hat{\lambda}=-0.0035$ and $\left(\begin{array}{l}\hat{a} \\ \hat{b}\end{array}\right)=\left(\begin{array}{c}-0.0587 \\ 4.0794\end{array}\right)$.

Then, the time response sequence function of 2017 is

$$
\hat{x}^{(1)}(t+1)=\left\{\left[\left(x^{(0)}(1)\right)^{(1+0.0035)}-\frac{4.0794}{-0.0587}\right] * e^{(1+0.0035) * 0.0587 * t}+\frac{4.0794}{-0.0587}\right\}^{\frac{1}{(1+0.0035)}}
$$

The different parameter values of each step indicate that the $\operatorname{RGPM}(\lambda, 1,1)$ can make the prediction result dynamic according to the characteristics of the input data. Fig.4 intuitively shows that the forecast results by $\operatorname{RGPM}(\lambda, 1,1)$ are in good agreement with the real values.

The prediction values of each grey model are shown in Table 4 and Fig.5,6. 
Table 4. Comparison of the original data and the predicted values of each model

\begin{tabular}{l|l|l|l|l|l|l}
\hline Year & $\begin{array}{l}\text { actual } \\
\text { value }\left(* 10^{12}\right)\end{array}$ & GM $(1,1)$ & GM $\left(1,1, x_{n}\right)$ & OICGM $(1,1)$ & NOGM $(1,1)$ & $\operatorname{RGPM}(\lambda, 1,1)$ \\
\hline 2008 & 3.45414 & 3.45414 & 3.45414 & 3.45414 & 3.45414 & 3.45414 \\
2009 & 3.70322 & 3.77109 & 3.94588 & 3.92903 & 4.0711 & 3.6663 \\
2010 & 4.19345 & 3.9498 & 4.23301 & 4.21493 & 4.3673 & 4.2270 \\
2011 & 4.70009 & 4.1371 & 4.54102 & 4.52163 & 4.6851 & 4.6620 \\
2012 & 4.97626 & 4.3332 & 4.87145 & 4.85064 & 5.0260 & 5.0223 \\
2013 & 5.42034 & 4.5386 & 5.22593 & 5.20360 & 5.317 & 5.3318 \\
2014 & 5.63837 & 4.7537 & 5.60619 & 5.58224 & 5.7840 & 5.6041 \\
2015 & 5.80200 & 4.97905 & 6.01413 & 5.988437 & 6.2049 & 5.8477 \\
2016 & 6.12971 & 5.21507 & 6.45175 & 6.424188 & 6.6564 & 6.0682 \\
2017 & 6.48210 & 5.46228 & 6.92121 & 6.891646 & 7.1408 & 6.4173 \\
\hline
\end{tabular}

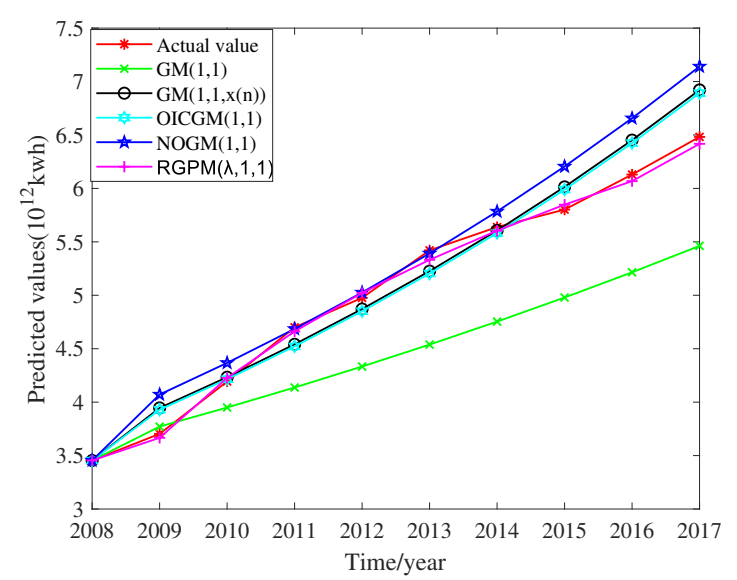

Fig. 5. Comparison of the original data and the predicted values of each model

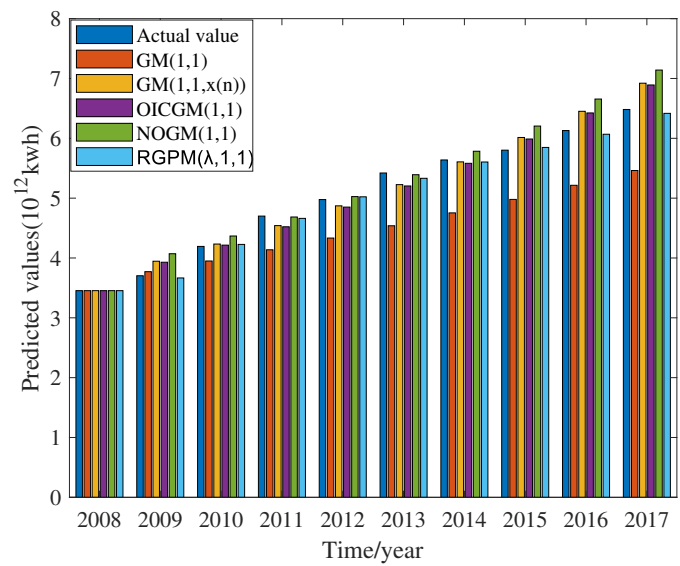

Fig. 6. The histogram of actual values and predicted values for each model

The related parameters and run time of each grey model are shown in Table 5.

Table 5. Related parameters and run time of each model

\begin{tabular}{c|c|c|c|c|c}
\hline parameter & GM $(1,1)$ & GM $\left(1,1, x_{n}\right)$ & OICGM $(1,1)$ & NOGM $(1,1)$ & $\operatorname{RGPM}(\lambda, 1,1)$ \\
\hline $\mathrm{a}$ & -0.07024 & -0.07024 & -0.07024 & -0.0702 & -0.0587 \\
\hline $\mathrm{b}$ & 3.56658 & 3.56658 & 3.56658 & 3.56658 & 4.0794 \\
\hline Run time & $0.044531 \mathrm{~s}$ & $0.0439114 \mathrm{~s}$ & $0.0439495 \mathrm{~s}$ & $0.0530092 \mathrm{~s}$ & $0.046502 \mathrm{~s}$ \\
\hline
\end{tabular}

Through Table 4 and Fig.5,6, it can be intuitively shown that the fitting degree between the predicted results of $\operatorname{RGPM}(\lambda, 1,1)$ and the real values is better than that of $\operatorname{GM}(1,1)$, $\operatorname{GM}\left(1,1, X_{n}\right), \operatorname{OICGM}(1,1)$ and $\operatorname{NOGM}(1,1)$. This result can also be verified by using the experimental test indicators mentioned in Section 3.6. The prediction effect and the detection results of each grey model are shown in Table 6. 
Table 6 . The absolute error and the inspection result of grey models

\begin{tabular}{c|c|c|c|c|c}
\hline Year & GM $(1,1)$ & GM $\left(1,1, x_{n}\right)$ & OICGM $(1,1)$ & $\operatorname{NOGM}(1,1)$ & $\operatorname{RGPM}(\lambda, 1,1)$ \\
\hline 2008 & 0 & 0 & 0 & 0 & 0 \\
2009 & 0.0679 & 0.2427 & 0.2258 & 0.3679 & 0.0369 \\
2010 & 0.2437 & 0.0396 & 0.0215 & 0.1738 & 0.0335 \\
2011 & 0.5630 & 0.1591 & 0.1785 & 0.0150 & 0.0381 \\
2012 & 0.6431 & 0.1048 & 0.1256 & 0.0497 & 0.0460 \\
2013 & 0.8817 & 0.1944 & 0.2167 & 0.0286 & 0.0885 \\
2014 & 0.8847 & 0.0322 & 0.0561 & 0.1456 & 0.0343 \\
2015 & 0.8229 & 0.2121 & 0.1864 & 0.4029 & 0.0457 \\
2016 & 0.9146 & 0.3220 & 0.2945 & 0.5267 & 0.0615 \\
2017 & 1.0198 & 0.4391 & 0.4095 & 0.6587 & 0.0648 \\
\hline MAE & 0.6041 & 0.1746 & 0.1715 & 0.2369 & $\mathbf{0 . 0 4 4 9}$ \\
\hline MAPE & $10.9339 \%$ & $3.2828 \%$ & $3.2260 \%$ & $4.4208 \%$ & $\mathbf{0 . 8 5 6 6} \%$ \\
\hline RMSE & 0.7006 & 0.2184 & 0.2094 & 0.3262 & $\mathbf{0 . 0 5 0 1}$ \\
\hline$r_{g}$ & 0.4991 & 0.7487 & 0.7490 & 0.7207 & $\mathbf{0 . 9 0 9 3}$ \\
\hline$P$ & 0.9000 & $\mathbf{1 . 0 0 0 0}$ & $\mathbf{1 . 0 0 0 0}$ & $\mathbf{1 . 0 0 0 0}$ & $\mathbf{1 . 0 0 0 0}$ \\
\hline$C$ & 0.3870 & 0.2100 & 0.2071 & 0.2487 & $\mathbf{0 . 1 2 8 2}$ \\
\hline
\end{tabular}

Through Table 6 , the values of 6 detection indexes by $\operatorname{GM}(1,1)$ are $M A E=0.6041$, $M A P E=10.9339 \%, R M S E=0.7006, r_{g}=0.4991, P=0.9000$ and $C=0.3870$. Since $M A P E=10.9339 \% \in[10 \%, 20 \%]$, it indicates that the prediction ability of $\mathrm{GM}(1,1)$ is weaker than other 4 grey prediction models. Moreover, its $P=0.9000<1.0000$, which further indicates that the predictive ability of $\operatorname{GM}(1,1)$ is weaker than that of $\operatorname{GM}\left(1,1, X_{n}\right)$, $\operatorname{OICGM}(1,1), \operatorname{NOGM}(1,1)$ and RGPM $(\lambda, 1,1)$.

In summary, $\operatorname{RGPM}(\lambda, 1,1)$ is superior to other four grey models in all detection indices. That is, its prediction performance is better than other four models. MAE and MAPE are increased by several percentage points respectively, indicating that the prediction accuracy of $\operatorname{RGPM}(\lambda, 1,1)$ is greatly improved compared with other four grey models. So, $\operatorname{RGPM}(\lambda, 1,1)$ is a better choice to analyze the forecast value of the country's total electricity consumption. However, the more accurate the prediction of national electricity consumption, the better it can provide more accurate forecast information for power workers. In order to improve the prediction performance of the grey power model, the weighted Markov model is used to modify it.

\subsection{The prediction results of $\operatorname{RGPMM}(\lambda, 1,1)$}

The $\operatorname{RGPM}(\lambda, 1,1)$ is used to get the fitted value $\hat{x}^{(0)}(t), t=1,2, \cdots, 8$ in each year, where 1-8 denote the 2008-2015, respectively. The accuracy index of grey fitting is obtained according to $Y(t)=\frac{x^{(0)}(t)}{\hat{x}^{(0)}(t)}$ presented in Section 3, as shown in Table 7.

Table 7. Grey fitting accuracy index and state division

\begin{tabular}{l|c|c|c|c|c|c|c|c}
\hline Year & 2008 & 2009 & 2010 & 2011 & 2012 & 2013 & 2014 & 2015 \\
\hline $\mathrm{Y}(\mathrm{t}) / \%$ & 100 & 95.7350 & 98.2257 & 101.1512 & 99.6787 & 102.2436 & 101.1984 & 99.9776 \\
\hline State & $E_{3}$ & $E_{1}$ & $E_{2}$ & $E_{4}$ & $E_{3}$ & $E_{5}$ & $E_{4}$ & $E_{3}$ \\
\hline
\end{tabular}

From Table 7, it can be found that the grey fitting accuracy index has a strong volatility, and the weighted Markov model can be used to predict the state of the grey fitting accuracy. 
The $Q$-type clustering (M et al., 2005) is used to divide the state. The clustering result is shown in Fig.7. According to the cluster diagram, the accuracy indices of grey fitting can be divided into 5 states, which are denoted as $\left\{E_{1}, E_{2}, E_{3}, E_{4}, E_{5}\right\}$. The clustering results are shown in Fig. 7 and Table 8. Then, the Markov state interval can be obtained as follows:

$$
\begin{aligned}
& E_{1} \in[95.7350 \%, 96.98035 \%), E_{2} \in[96.98035 \%, 99.5000 \%), E_{3} \in[99.5000 \%, 101.0000 \%), \\
& E_{4} \in[101.0000 \%, 101.7210 \%), E_{5} \in[101.7210 \%, 102.2500 \%)
\end{aligned}
$$

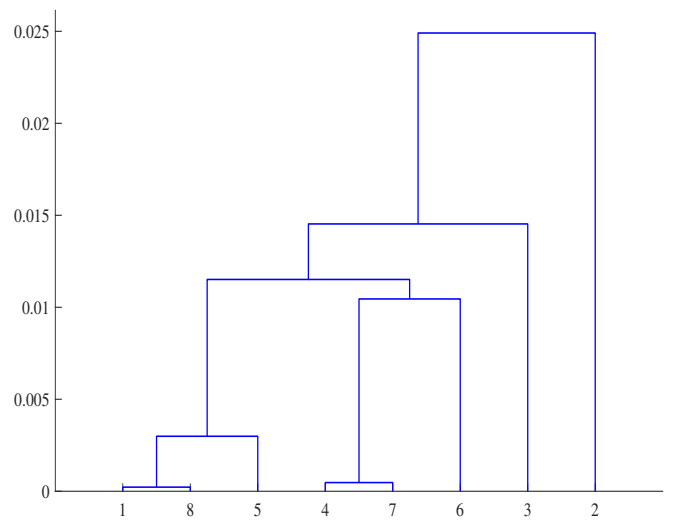

Fig. 7. The figure of Q-cluster for 2008-2015

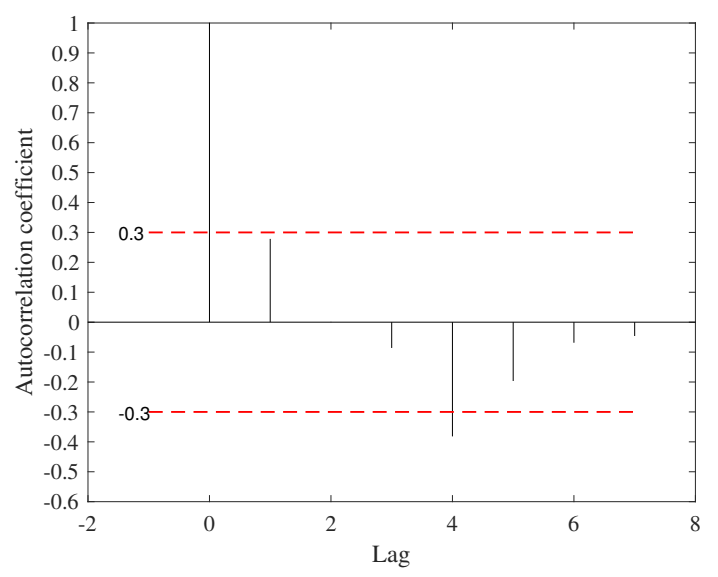

Fig. 8. The each order of autocorrelation coefficient

According to Fig.9, it is found that when $\mathrm{m}$ is taken as 4, the condition of $\left|r_{\omega}\right| \geq 0.3$ is satisfied, and there are $r_{1}=0.2306, r_{2}=-0.0694, r_{3}=-0.0412, r_{4}=-0.4134$. Then the Markov weights of each order are obtained as follows:

$$
\begin{aligned}
& \theta_{1}=\frac{0.2306}{0.2306+0.0694+0.0412+0.4134}=0.3056, \theta_{2}=\frac{0.0694}{0.2306+0.0694+0.0412+0.4134}=0.0920, \\
& \theta_{3}=\frac{0.0412}{0.2306+0.0694+0.0412+0.4134}=0.0546, \theta_{4}=\frac{0.4134}{0.2306+0.0694+0.0412+0.4134}=0.5478 .
\end{aligned}
$$

The Markov transition probability matrixes of each step are calculated as follows: 


$$
\begin{aligned}
& p(1)=\left(\begin{array}{ccccc}
0 & 1 & 0 & 0 & 0 \\
0 & 0 & 0 & 1 & 0 \\
0.5 & 0 & 0 & 0 & 0.5 \\
0 & 0 & 1 & 0 & 0 \\
0 & 0 & 0 & 1 & 0
\end{array}\right), \quad p(2)=\left(\begin{array}{ccccc}
0 & 0 & 0 & 1 & 0 \\
0 & 0 & 1 & 0 & 0 \\
0 & 0.5 & 0 & 0.5 & 0 \\
0 & 0 & 0 & 0 & 1 \\
0 & 0 & 1 & 0 & 0
\end{array}\right), \\
& p(3)=\left(\begin{array}{ccccc}
0 & 0 & 1 & 0 & 0 \\
0 & 0 & 0 & 0 & 1 \\
0 & 0 & 0.5 & 0.5 & 0 \\
0 & 0 & 0 & 1 & 0 \\
0 & 0 & 0 & 0 & 0
\end{array}\right), \quad p(4)=\left(\begin{array}{lllll}
0 & 0 & 0 & 0 & 1 \\
0 & 0 & 0 & 1 & 0 \\
0 & 0 & 1 & 0 & 0 \\
0 & 0 & 1 & 0 & 0 \\
0 & 0 & 0 & 0 & 0
\end{array}\right) \text {. }
\end{aligned}
$$

The accuracy index of grey fitting is in the state of $E_{3}$ in 2015 , so we can get:

The one-step transition probability vector is $(0.5,0,0,0,0.5)$, and the corresponding Markov weight is $\theta_{1}=0.3056$;

The two-step transition probability vector is $(0,0.5,0,0.5,0)$, and the Markov weight is $\theta_{2}=0.0920$;

The three-step transition probability vector is $(0,0,0.5,0.5,0)$, and the Markov weight is $\theta_{3}=0.0546$

The four-step transition probability vector is $(0,0,1,0,0)$, and the Markov weight is $\theta_{4}=$ 0.5478 .

According to $p_{i}=\sum_{\omega=1}^{m} \theta_{\omega} p_{i}(\omega)$, the weighted Markov prediction probability is calculated, as shown in Table 9 .

Table 9. Calculation results of weighted Markov transition probability

\begin{tabular}{c|c|c|c|c|c|c|c}
\hline start year & state & weight & $E_{1}$ & $E_{2}$ & $E_{3}$ & $E_{4}$ & $E_{5}$ \\
\hline 2015 & $E_{3}$ & 0.3056 & 0.5 & 0 & 0 & 0 & 0.5 \\
\hline 2014 & $E_{4}$ & 0.0920 & 0 & 0.5 & 0 & 0.5 & 0 \\
\hline 2013 & $E_{5}$ & 0.0546 & 0 & 0 & 0.5 & 0.5 & 0 \\
\hline 2012 & $E_{3}$ & 0.5478 & 0 & 0 & 1 & 0 & 0 \\
\hline
\end{tabular}

From Table 9, the weighted probability value of the transition probability can be obtained. Based on results, $\max \left\{p_{i}\right\}=p_{3}=0.5751$, that is, the probability of being in the state of $E_{3}$ in 2016 is the largest. And its adjacent states are $E_{2}$ and $E_{4}$. According to Formula (15), perform linear interpolation on the interval of state $E_{3}$, and the predicted value of grey fitting accuracy is

$$
\widehat{Y}(9)=\otimes_{1 i} \times \frac{0.0460}{0.0460+0.0733}+\otimes_{2 i} \times \frac{0.0733}{0.0460+0.0733}=100.4300 \% .
$$

Through the correction of the grey fitting accuracy index, the more accurate predicted value in 2016 can be obtained as follows:

$$
\widetilde{x}^{(0)}(9)=\hat{x}^{(0)}(9) * \widehat{Y}(9)=6.0682 \times 100.4300 \%=6.0943
$$

make mupdeling process the introduction of rolling meghanism makes the established model using the latest data from 2009 to 2016, the proposed $\operatorname{RGPMM}(\lambda, 1,1)$ model can be built to obtain the predicted value in 2017. The results are as follows: 
The grey fitting accuracy index in 2017 is in Table 10 .

Table 10. Grey fitting accuracy index of 2017

\begin{tabular}{l|c|c|c|c|c|c|c|c}
\hline Year & 2009 & 2010 & 2011 & 2012 & 2013 & 2014 & 2015 & 2016 \\
\hline $\mathrm{Y}(\mathrm{t}) / \%$ & 100 & 97.3600 & 100.5900 & 99.8000 & 102.6600 & 101.3300 & 99.2300 & 99.9900 \\
\hline
\end{tabular}
10 .

The clustering result and the autocorrelation diagram are shown in Table 11 and Fig.9,

Table 11. The correlation coefficients $(r)$ and Q-cluster results during 2009-2016

\begin{tabular}{c|c|c|c|c|c|c|c|c}
\hline Label & 1 & 2 & 3 & 4 & 5 & 6 & 7 & 8 \\
\hline Year & 2009 & 2010 & 2011 & 2012 & 2013 & 2014 & 2015 & 2016 \\
\hline$r$ & 1.0000 & 0.0104 & -0.0471 & -0.3873 & -0.2401 & 0.1349 & 0.0282 & 0.0009 \\
\hline \multicolumn{8}{c}{ Class 1: 2015} \\
Q-cluster:
\end{tabular}

Class 3: 2011

Class 4: 2014

Class 5: 2013

Class 6: 2010

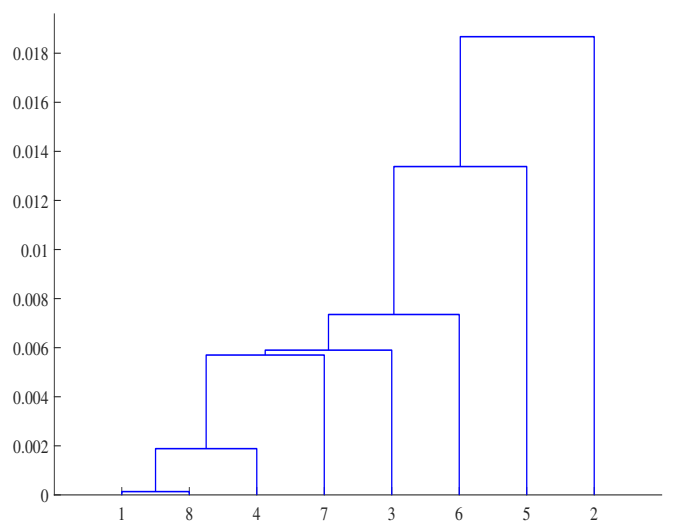

Fig. 9. The figure of Q-cluster for 2008-2015

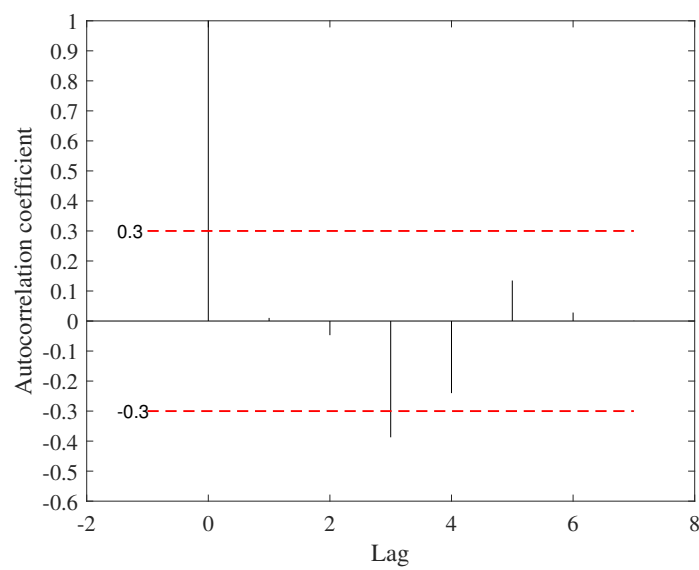

Fig. 10. The each order of autocorrelation coefficient

After the correction by Markov blanket, the more accurate prediction value in 2017 is 6.5258

4.4. Comparing the forecasting performance of $\operatorname{RGPM}(\lambda, 1,1)$ and $\operatorname{RGPMM}(\lambda, 1,1)$

The predicted performance of $\operatorname{RGPMM}(\lambda, 1,1)$ model is compared with the $\operatorname{RGPM}(\lambda, 1,1)$ model. MSE and MAPE are selected to evaluate the predictive performance of the models, and their expressions are respectively as follows:

$$
M S E=\frac{1}{2} * \sum_{i=9}^{10}\left(x_{i}-\hat{x}_{i}\right)^{2}, M A P E=\frac{1}{2} * \sum_{i=9}^{10}\left|\frac{x_{i}-\hat{x}_{i}}{x_{i}}\right| \times 100 \%
$$

MSE and MAPE of each model are calculated. The result is in Table 12.

Table 12. Comparison of prediction effects from the models

\begin{tabular}{c|c|c|c|c}
\hline model & 2016 & 2017 & MSE & MAPSE \\
\hline true value $\left(10^{12}\right)$ & 6.12971 & 6.48210 & $/$ & $/$ \\
\hline RGPM $(1,1)$ & 6.0682 & 6.4173 & 0.0040 & $1.00 \%$ \\
\hline RGPMM $(1,1)$ & 6.0943 & 6.5258 & $\mathbf{0 . 0 0 1 6}$ & $\mathbf{0 . 6 3 \%}$ \\
\hline
\end{tabular}


In Table 12 , it is found that the values of MSE and MAPE by $\operatorname{RGPMM}(\lambda, 1,1)$ are smaller than $\operatorname{RGPM}(\lambda, 1,1)$. The result shows that the $\operatorname{RGPMM}(\lambda, 1,1)$ further improves the accuracy.

\subsection{Forecast the total electricity consumption in the next 6 years}

Because that $\operatorname{RGPMM}(\lambda, 1,1)$ has been shown to provide accurate prediction, we use it to forecast China's electricity consumption from 2018 to 2023. During the process, the results by Q-cluster and the correlation coefficients of each year are in Table 13-18 and Fig.11-22.

Table 13. The correlation coefficients $(r)$ and Q-cluster results during 2010-2017

\begin{tabular}{c|c|c|c|c|c|c|c|c}
\hline Label & 1 & 2 & 3 & 4 & 5 & 6 & 7 & 8 \\
\hline Year & 2010 & 2011 & 2012 & 2013 & 2014 & 2015 & 2016 & 2017 \\
\hline$r$ & 1.0000 & 0.2228 & -0.5766 & -0.3175 & 0.0345 & 0.0842 & 0.0527 & 0.0000 \\
\hline \multirow{3}{*}{ Q-cluster: } & Class 1: 2013 & \multicolumn{1}{l}{ Class 2: 2014} \\
& Class 3: 2011 & & & & \\
& Class 4: $2010,2012,2015,2016,2017$ \\
\hline
\end{tabular}

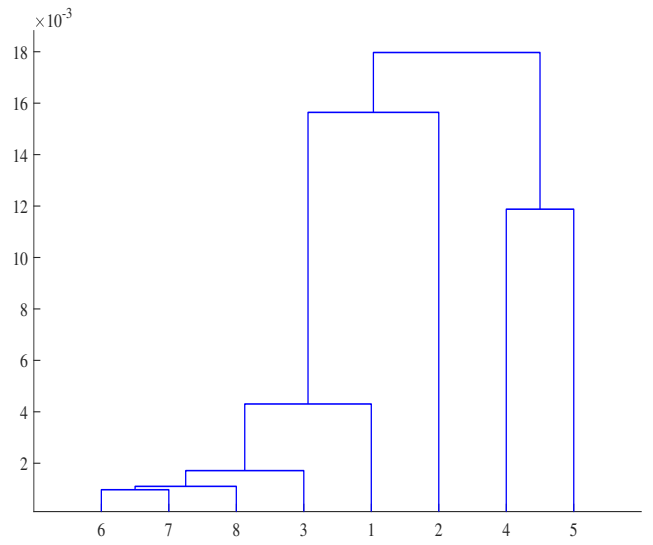

Fig. 11. The figure of Q-cluster during 2010-2017

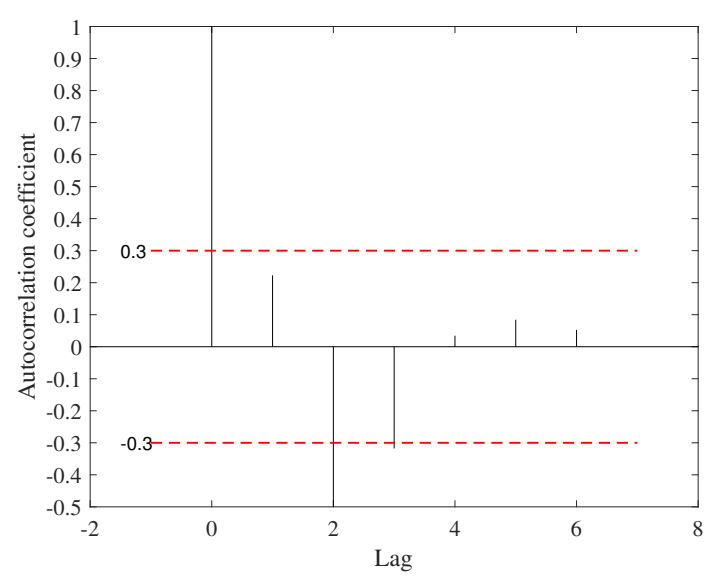

Fig. 12. The each order of autocorrelation coefficient for 2018

Table 14. The correlation coefficients $(r)$ and Q-cluster results during 2011-2018

\begin{tabular}{c|c|c|c|c|c|c|c|c}
\hline Label & 1 & 2 & 3 & 4 & 5 & 6 & 7 & 8 \\
\hline Year & 2011 & 2012 & 2013 & 2014 & 2015 & 2016 & 2017 & 2018 \\
\hline$r$ & 1.0000 & -0.1076 & -0.3212 & -0.0884 & -0.0879 & 0.0363 & 0.0686 & 0.0003 \\
\hline \multirow{3}{*}{ Q-cluster: } & Class 1: 2013,2014 & Class 2: $2011,2015,2016,2017,2018$ \\
& Class 3: 2012
\end{tabular}




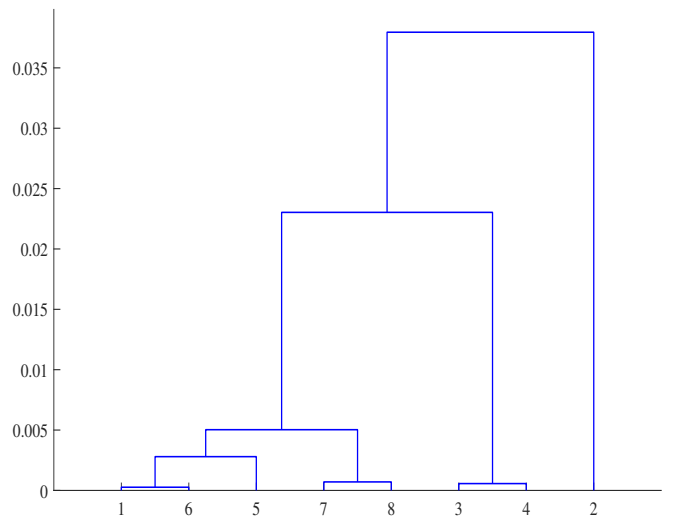

Fig. 13. The figure of Q-cluster during 2011-2018

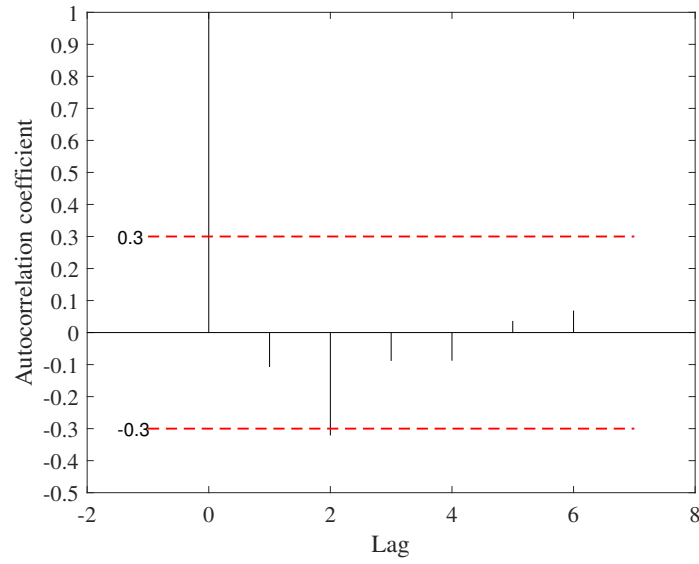

Fig. 14. The each order of autocorrelation

Table 15. The correlation coefficients $(r)$ and Q-cluster results during 2012-2019

\begin{tabular}{c|c|c|c|c|c|c|c|c}
\hline Label & 1 & 2 & 3 & 4 & 5 & 6 & 7 & 8 \\
\hline Year & 2012 & 2013 & 2014 & 2015 & 2016 & 2017 & 2018 & 2019 \\
\hline$r$ & 1.0000 & -0.4445 & 0.0280 & -0.1651 & 0.0121 & 0.1111 & -0.0428 & 0.0012 \\
\hline \multirow{5}{*}{ Q-cluster: } & Class 1: 2017,2018 & Class 2: $2012,2015,2016,2019$ & & & \\
& Class 3: 2014 & & & & \\
& Class 4: 2013
\end{tabular}

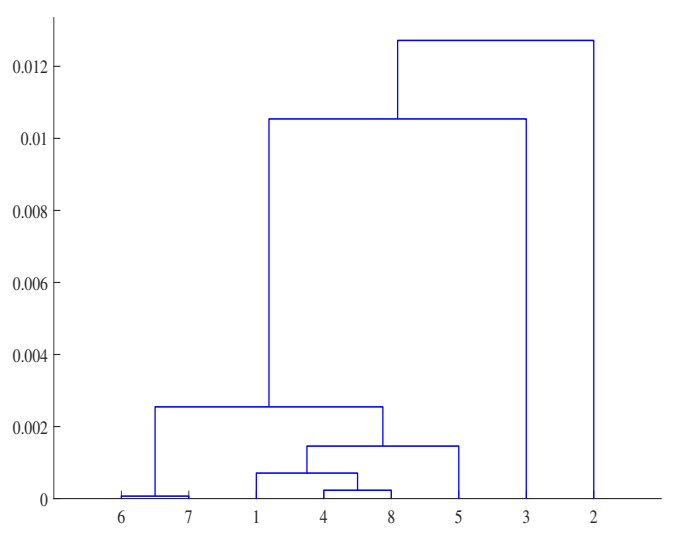

Fig. 15. The figure of Q-cluster during 2012-2019

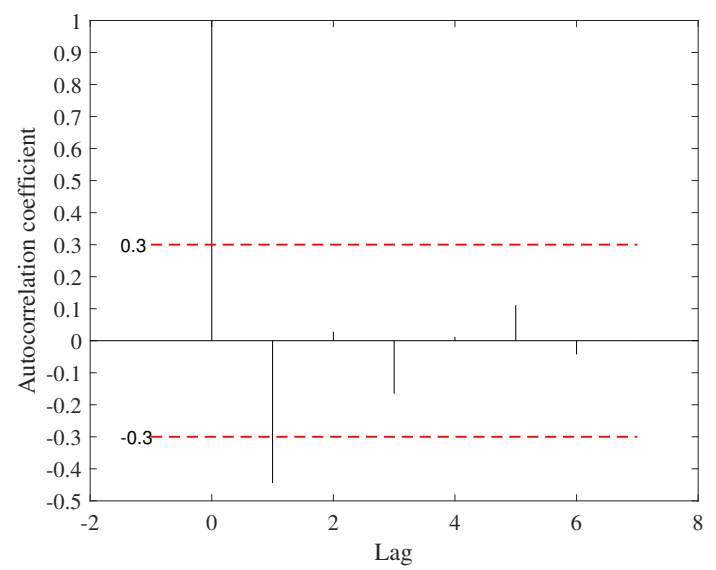

Fig. 16. The each order of autocorrelation coefficient of 2020

Table 16. The correlation coefficients $(r)$ and Q-cluster results during 2013-2020

\begin{tabular}{c|c|c|c|c|c|c|c|c}
\hline Label & 1 & 2 & 3 & 4 & 5 & 6 & 7 & 8 \\
\hline Year & 2013 & 2014 & 2015 & 2016 & 2017 & 2018 & 2019 & 2020 \\
\hline$r$ & 1.0000 & -0.2805 & -0.4265 & 0.2937 & 0.0960 & -0.1968 & -0.0145 & 0.0286 \\
\hline & Class 1: 2013 \\
& Class 2: 2019,2020 \\
Q-cluster: & Class 3: 2014 & & & & & \\
& Class 4: $2015,2017,2018$ \\
& Class 5: 2016
\end{tabular}




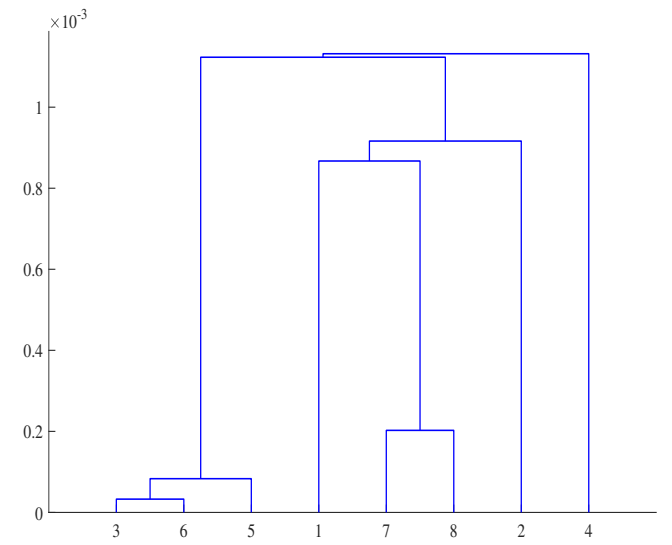

Fig. 17. The figure of Q-cluster during 2013-2020

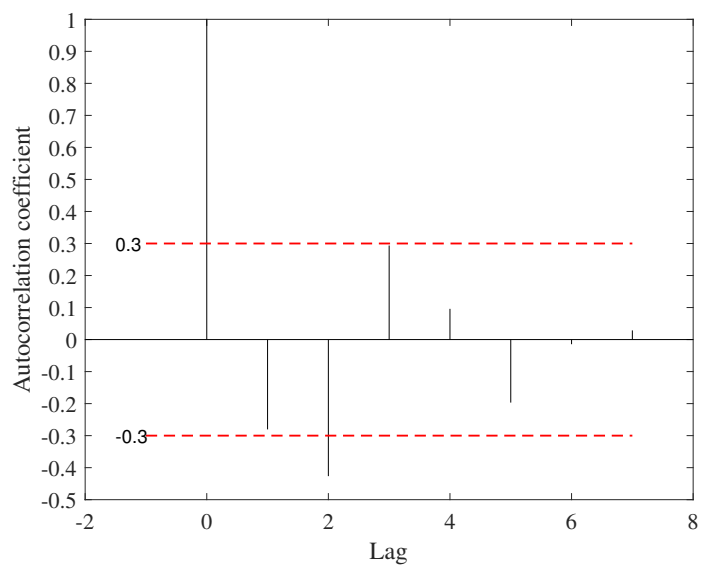

Fig. 18. The each order of autocorrelation coefficient for 2021

Table 17. The correlation coefficients $(r)$ and Q-cluster results during 2014-2021

\begin{tabular}{c|c|c|c|c|c|c|c|c}
\hline Label & 1 & 2 & 3 & 4 & 5 & 6 & 7 & 8 \\
\hline Year & 2014 & 2015 & 2016 & 2017 & 2018 & 2019 & 2020 & 2021 \\
\hline$r$ & 1.0000 & -0.4712 & -0.0961 & 0.2323 & -0.0760 & -0.1330 & 0.0445 & -0.0005 \\
\hline \multirow{5}{*}{ Q-cluster: } & Class 1: 2019,2020 & Class 2: $2014,2017,2018,2021$ & & & \\
& Class 3: 2016 & & & & & \\
& Class 4: 2015
\end{tabular}

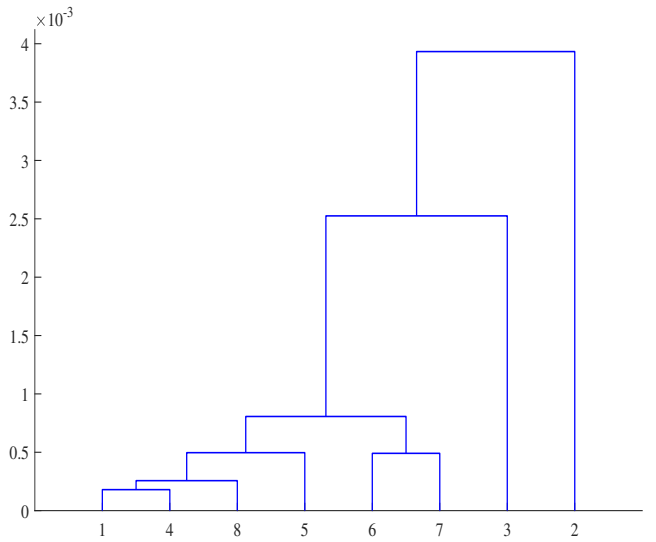

Fig. 19. The figure of Q-cluster during 2014-2021

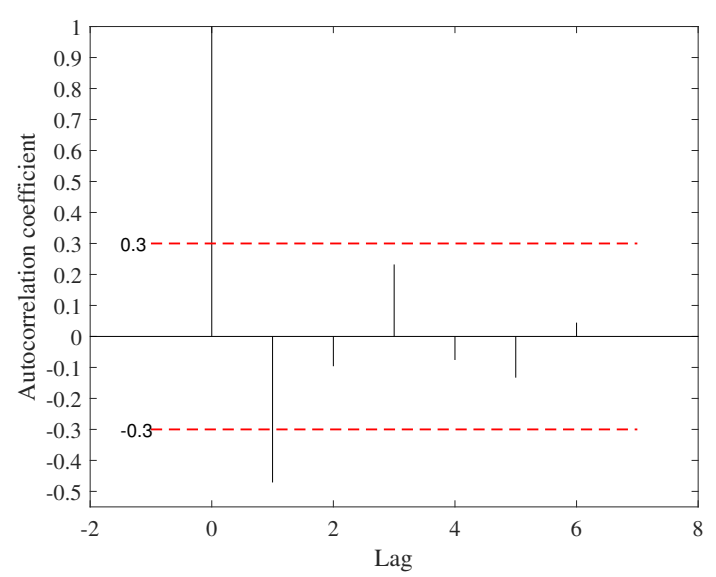

Fig. 20. The each order of autocorrelation coefficient for 2022

Table 18. The correlation coefficients $(r)$ and Q-cluster results during 2015-2022

\begin{tabular}{c|c|c|c|c|c|c|c|c}
\hline Label & 1 & 2 & 3 & 4 & 5 & 6 & 7 & 8 \\
\hline Year & 2015 & 2016 & 2017 & 2018 & 2019 & 2020 & 2021 & 2022 \\
\hline$r$ & 1.0000 & 0.2038 & -0.4346 & -0.1974 & -0.0552 & -0.0086 & -0.0074 & -0.0006 \\
\hline \multirow{5}{*}{ Q-cluster: } & Class 1: 2016,2021 & Class 2: 2015,2022 & & & & \\
& Class 3: 2019,2020 & & & & \\
& Class 4: 2017,2018
\end{tabular}




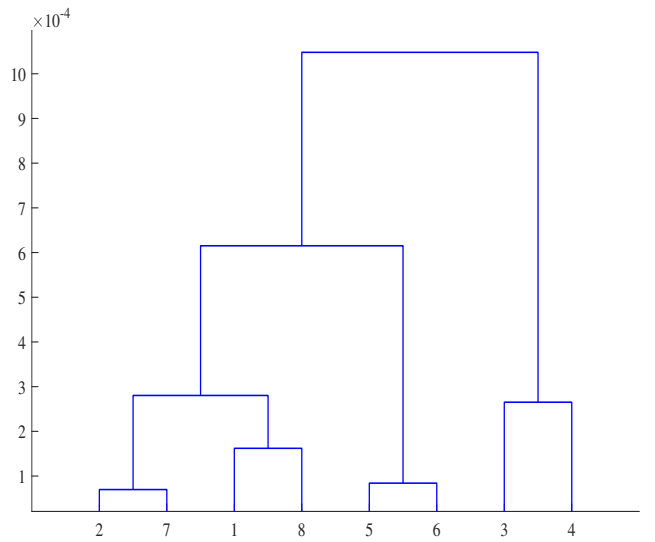

Fig. 21. The figure of Q-cluster during 2015-2022

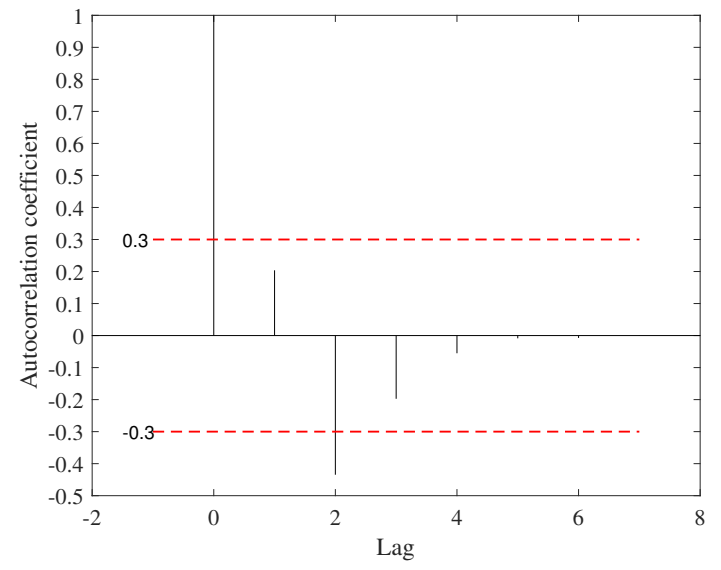

Fig. 22. The each order of autocorrelation coefficient for 2023

The prediction results are modified by Markov theory during 2018-2023 are shown in Fig.23-28.
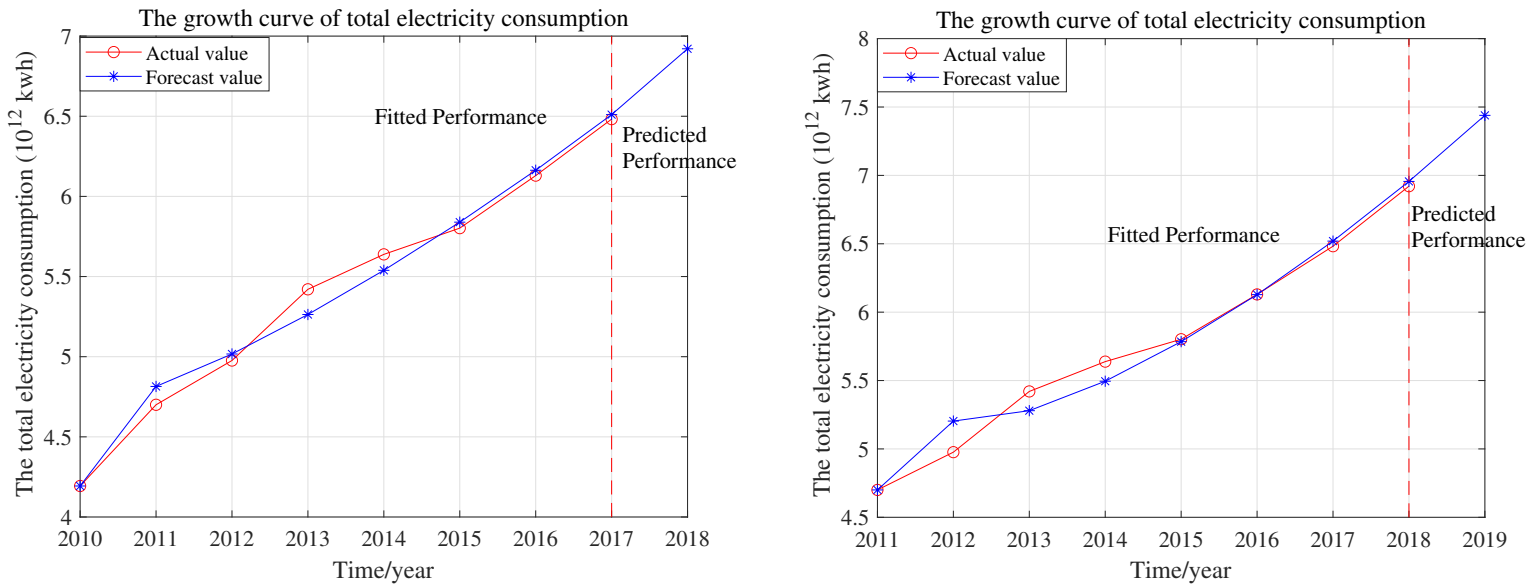

Fig. 23. The predicted value of $\operatorname{RGPMM}(\lambda, 1,1)$ for $\operatorname{Fig}$. 24. The predicted value of $\operatorname{RGPMM}(\lambda, 1,1)$ for 2018
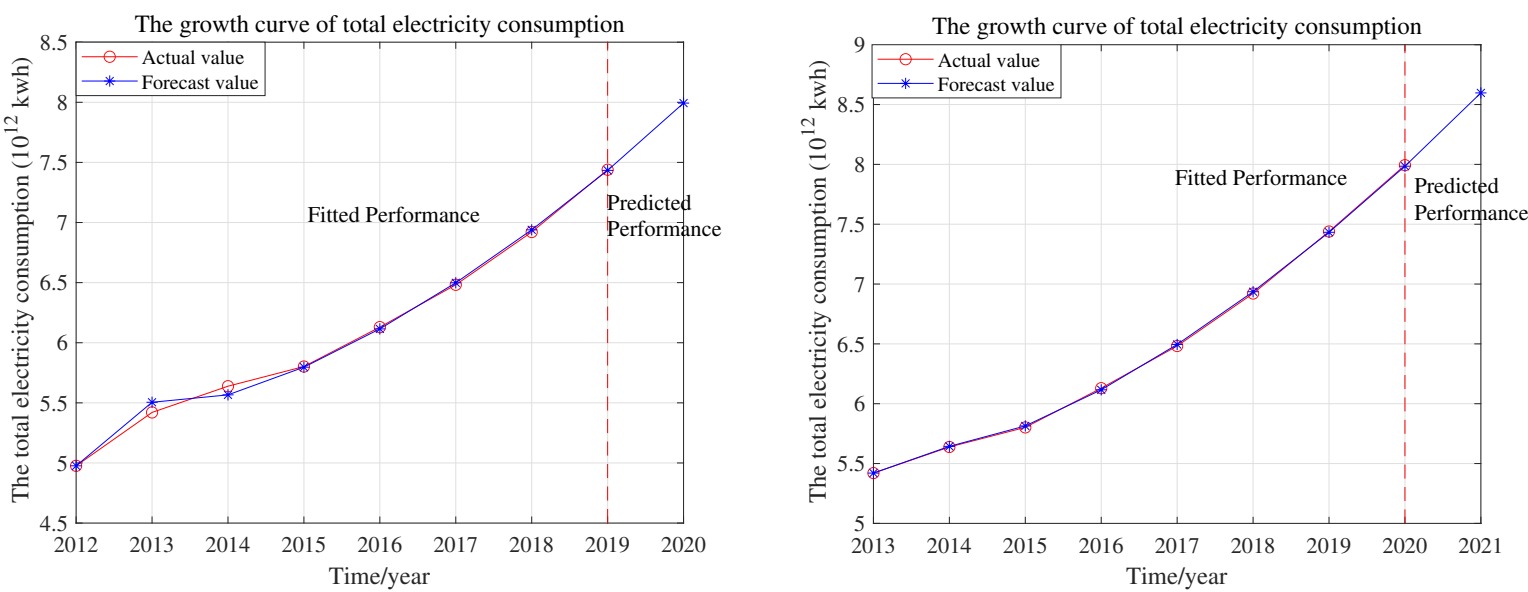

Fig. 25. The predicted value of $\operatorname{RGPMM}(\lambda, 1,1)$ for Fig. 26. The predicted value of $\operatorname{RGPMM}(\lambda, 1,1)$ for 2020 2021 

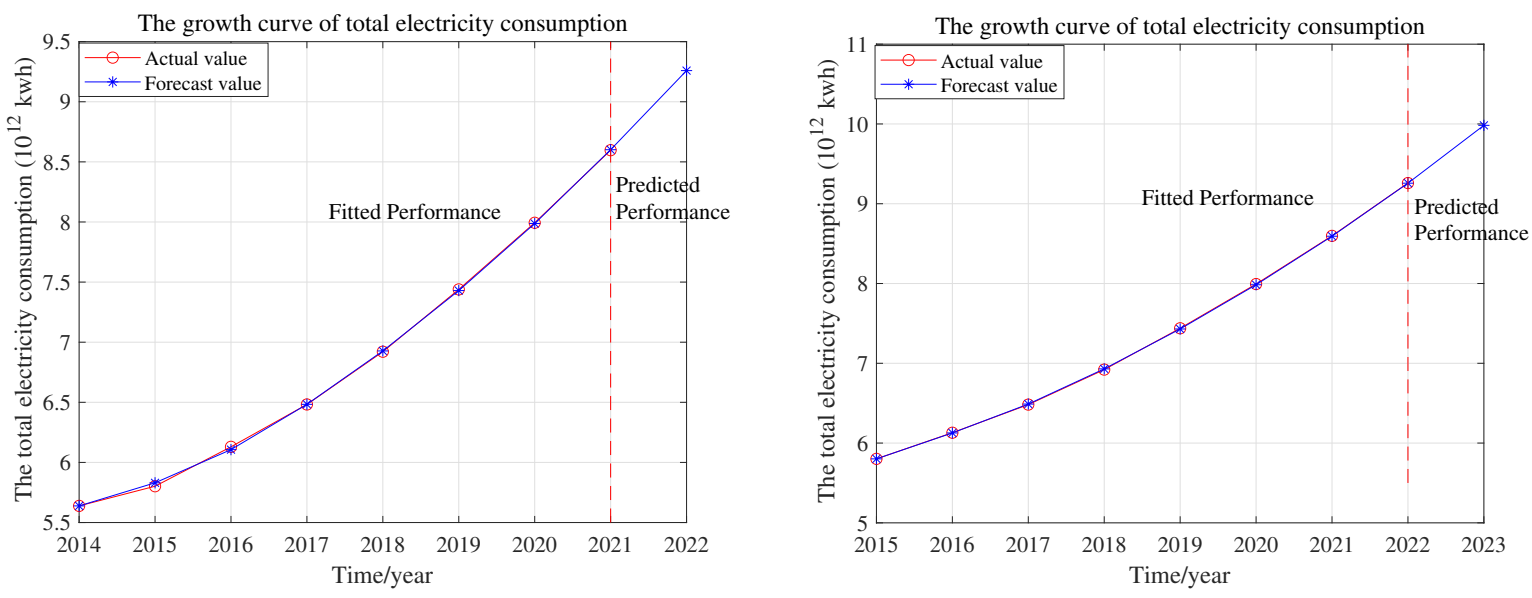

Fig. 27. The predicted value of $\operatorname{RGPMM}(\lambda, 1,1)$ for Fig. 28. The predicted value of $\operatorname{RGPMM}(\lambda, 1,1)$ for 2022 2023

The parameters and forecasted results are presented in Table 19.

Table 19. The predicted values $\left(10^{12} \mathrm{kwh}\right)$ and the model parameters of $\operatorname{RGPMM}(\lambda, 1,1)$ during 2018-2023

\begin{tabular}{c|c|c|c|c|c|c}
\hline Year & 2018 & 2019 & 2020 & 2021 & 2022 & 2023 \\
\hline $\mathrm{a}$ & -0.0587 & -0.0774 & -0.0827 & -0.0824 & -0.0816 & -0.0792 \\
\hline $\mathrm{b}$ & 4.7181 & 6.0109 & 6.6204 & 6.3135 & 6.044 & 5.9985 \\
\hline$\lambda$ & -0.0341 & -0.132 & -0.1525 & -0.1152 & -0.0769 & -0.0464 \\
\hline predicted value & 6.9209 & 7.4390 & 7.9934 & 8.5973 & 9.2592 & 9.9826 \\
\hline
\end{tabular}

It can be seen from experimental results in Table 19 that the total electricity consumption will maintain a growth trend. It is estimated that by 2023, electricity consumption will increase from $6.48210 * 10^{12} \mathrm{kwh}$ in 2017 to $9.9826 * 10^{12} \mathrm{kwh}$, which is nearly $10^{13} \mathrm{kwh}$, almost twice that of 2012. This prediction result is of great significance to energy planning and policy-making. In order to ensure secure and stable energy and power supply, it is necessary to accelerate the establishment and improvement of auxiliary services for the power allocation, continuously improve the capacity of power system, and ensure the perfection of power infrastructure in the supply chain. Meanwhile, it is worth noting that huge consumption will bring a heavy burden to energy planning and environmental protection. Therefore, the government needs to take appropriate actions and plans to meet the high energy demand in the future, so as to avoid the phenomenon of resource waste caused by unreasonable resource arrangement.

\section{Conclusion}

Electricity consumption forecast is of great significance to the economic development and the guarantee of people's life. However, due to the increase in electricity demand and in the complexity of the power system, it is becoming more and more difficult to accurately predict the power consumption. Therefore, it is meaningful to design a prediction method which is suitable for the limited data.

Aiming at the problem of power demand under limited data, a grey power-Markov forecasting model based on rolling mechanism is proposed. It predicts the electricity consump- 
tion on the basis of grey theory by introducing rolling mechanism and Markov state prediction.According to the research results, the following conclusions can be drawn:

1) In view of the problems faced by power system load forecasting, $\operatorname{RGPMM}(\lambda, 1,1)$ is proposed. The example shows that it gets a better prediction effect than the traditional $\operatorname{GM}(1,1)$ models, and it improves the prediction accuracy to a certain extent. This model provides a new way to forecast electricity consumption. The structure of the new grey prediction model is simple, the modeling process is easy to operate, and it is easy to be applied in other fields.

2)Total electricity consumption is not only an important indicator of economic development, but also an important indicator of formulating the energy strategy and related environmental protection policies. The forecast results of the total electricity consumption indicate that China's total power consumption will continue to maintain a strong growth trend in the next few years. It has certain reference value for formulating the energy strategy and related environmental protection policies.

Future work: The forecasting model proposed in this article is for univariate, and multivariate gray models can be considered in the future. In addition, the new model proposed in this paper can also be used to forecast the industrial electricity consumption, the agricultural electricity consumption, the residential electricity consumption and other fields.

\section{Acknowledgments}

The authors are grateful to the editor and reviewers for their valuable comments. This work is financially supported by the National Natural Science Foundation of China (61573266) and Natural Science Basic Research Program of Shaanxi(Program No.2021JM-133).

\section{Declarations}

-Ethical Approval

NOT Applicable

-Consent to Participate

NOT Applicable

-Consent to Publish

NOT Applicable

-Authors Contributions

Liqin Sun contributed the idea. Liqin Sun and Youlong Yang performed the calculations. Jiadi Zhu checked the calculations. Liqin Sun wrote the main manuscript, Youlong Yang and Tong Ning made an improvement. All authors contributed to discussion and reviewed the manuscript.

-Funding

This work is financially supported by the National Natural Science Foundation of China (61573266) and Natural Science Basic Research Program of Shaanxi(Program No.2021JM133).

-Competing Interests

The authors declare that they have no known competing financial interests or personal relationships that could have appeared to influence the work reported in this paper.

-The data in this article are from 
The data in this article are from: "http://www.stats.gov.cn/tjsj/ndsj/2019/indexch.htm".

\section{References}

Azadeh A, Ghaderi S F, Sohrabkhani S. A simulated-based neural network algorithm for forecasting electrical energy consumption in Iran[J]. Energy policy, 2008, 36(7): 2637-2644. Akay D, Atak M. Grey prediction with rolling mechanism for electricity demand forecasting of Turkey[J]. energy, 2007, 32(9): 1670-1675.

Amber K P, Ahmad R, Aslam M W, et al. Intelligent techniques for forecasting electricity consumption of buildings[J]. Energy, 2018, 157: 886-893.

Akdi Y, Gölveren E, Okkaoğlu Y. Daily electrical energy consumption: Periodicity, harmonic regression method and forecasting[J]. Energy, 2020, 191: 116524.

Bekiroglu K, Duru O, Gulay E, et al. Predictive analytics of crude oil prices by utilizing the intelligent model search engine[J]. Applied Energy, 2018, 228: 2387-2397.

Bahrami S, Hooshmand R A, Parastegari M. Short term electric load forecasting by wavelet transform and grey model improved by PSO (particle swarm optimization) algorithm[J]. Energy, 2014, 72: 434-442.

Chui F, Elkamel A, Surit R, et al. Long-term electricity demand forecasting for power system planning using economic, demographic and climatic variables[J]. European Journal of Industrial Engineering, 2009, 3(3): 277-304.

CAO Jian, SHI Shiliang, CAO Huajuan, et al. Study on prediction and relationship of road transportation accidents of dangerous chemicals and traffic accidents based on GM (1, 1)-Markov model[J]. Journal of Safety Science and Technology, 2019, 15(1): 26-31.

China Statistical Yearbook of 2019.

Ding S, Hipel K W, Dang Y. Forecasting China's electricity consumption using a new grey prediction model[J]. Energy, 2018, 149: 314-328.

Dang Y G, Liu S F, Liu B. The GM models that x(n) be taken as initial value[J]. Kybernetes, 2004, 33(2): 247-254.

Guo H, Xiao X, Forrest J. A research on a comprehensive adaptive grey prediction model CAGM $(1, \mathrm{~N})[\mathrm{J}]$. Applied Mathematics and Computation, 2013, 225: 216-227.

Ghani I M M, Ahmad S. Stepwise multiple regression method to forecast fish landing[J]. Procedia-Social and Behavioral Sciences, 2010, 8: 549-554.

Geem Z W, Roper W E. Energy demand estimation of South Korea using artificial neural network[J]. Energy policy, 2009, 37(10): 4049-4054.

Hussain A, Rahman M, Memon J A. Forecasting electricity consumption in Pakistan: The way forward[J]. Energy Policy, 2016, 90: 73-80.

Hamzaçebi C. Forecasting of Turkey's net electricity energy consumption on sectoral bases $[\mathrm{J}]$. Energy policy, 2007, 35(3): 2009-2016.

Hernández L, Baladrón C, Aguiar J M, et al. Experimental analysis of the input variables; ${ }^{-}$ relevance to forecast next dayi ${ }^{-} \mathrm{s}$ aggregated electric demand using neural networks $[\mathrm{J}]$. Energies, 2013, 6(6): 2927-2948.

Hernandez L, Baladron C, Aguiar J M, et al. A survey on electric power demand forecasting: future trends in smart grids, microgrids and smart buildings[J]. IEEE Communications Surveys \& Tutorials, 2014, 16(3): 1460-1495. 
HE Yong, BAO Yidan. Grey-Markov forecasting model and its application[J]. Systems Engineering; ${ }^{\text {a }}$ Theory \& Practice, 1992,12(4): 59-63.

Julong D. Grey control system[J]. Journal of Huazhong University of Science and Technology, 1982, 3(9): 18.

Julong D. Introduction to grey system theory[J]. The Journal of grey system, 1989, 1(1): $1-24$.

Khosravi A, Nahavandi S, Creighton D, et al. Interval type-2 fuzzy logic systems for load forecasting: A comparative study[J]. IEEE Transactions on Power Systems, 2012, 27(3): 1274-1282.

Kavousi-Fard A, Samet H, Marzbani F. A new hybrid modified firefly algorithm and support vector regression model for accurate short term load forecasting[J]. Expert systems with applications, 2014, 41(13): 6047-6056.

Kumar U, Jain V K. Time series models (Grey-Markov, Grey Model with rolling mechanism and singular spectrum analysis) to forecast energy consumption in India[J]. Energy, 2010, 35(4): 1709-1716.

Lin B, Liu C. Why is electricity consumption inconsistent with economic growth in China?[J]. Energy Policy, 2016, 88: 310-316.

Liu T, Wang B, Yang C. Online Markov Chain-based energy management for a hybrid tracked vehicle with speedy Q-learning[J]. Energy, 2018, 160: 544-555.

Lin Z, Zhang Q, Liu H. Parameters optimization of GM $(1,1)$ model based on artificial fish swarm algorithm[J]. Grey Systems: Theory and Application, 2012.

Mohamed Z, Bodger P. Forecasting electricity consumption in New Zealand using economic and demographic variables[J]. Energy, 2005, 30(10): 1833-1843.

Narasimhan M, Jojic N, Bilmes J A. Q-clustering[J]. Advances in Neural Information Processing Systems, 2005, 18: 979-986.

Pai T Y, Chiou R J, Wen H H. Evaluating impact level of different factors in environmental impact assessment for incinerator plants using $\operatorname{GM}(1, \mathrm{~N})$ model[J]. Waste Management, 2008, 28(10): 1915-1922.

Tien T L. The indirect measurement of tensile strength for a higher temperature by the new model IGDMC (1, n)[J]. Measurement, 2008, 41(6): 662-675.

Wang J, Zhu S, Zhao W, et al. Optimal parameters estimation and input subset for grey model based on chaotic particle swarm optimization algorithm[J]. Expert Systems with Applications, 2011, 38(7): 8151-8158.

Wang J, Zhou Q, Zhang X. Wind power forecasting based on time series ARMA model[C] \\ IOP Conference Series: Earth and Environmental Science. IOP Publishing, 2018, 199(2): 022015.

Wang S, Wang P, Zhang Y. A prediction method for urban heat supply based on grey system theory[J]. Sustainable Cities and Society, 2020, 52: 101819.

Wang Z X, Dang Y G, Pei L L. Modeling approach for oscillatory sequences based on GM(1, 1) power model[J]. Systems Engineering and Electronics, 2011, 33(11): 2440-2444.

Xiong P, Dang Y, Yao T, et al. Optimal modeling and forecasting of the energy consumption and production in China[J]. Energy, 2014, 77: 623-634.

Xiao X, Yang J, Mao S, et al. An improved seasonal rolling grey forecasting model using a cycle truncation accumulated generating operation for traffic flow[J]. Applied Mathematical Modelling, 2017, 51: 386-404. 
Zeng B, Ma X, Shi J. Modeling method of the grey $\operatorname{GM}(1,1)$ model with interval grey action quantity and its application[J]. Complexity, 2020, 2020.

Zhang Y, Chen B, Pan G, et al. A novel hybrid model based on VMD-WT and PCABP-RBF neural network for short-term wind speed forecasting $[\mathrm{J}]$. Energy Conversion and Management, 2019, 195: 180-197.

Zhao W, Wang J, Lu H. Combining forecasts of electricity consumption in China with timevarying weights updated by a high-order Markov chain model[J]. Omega, 2014, 45: 80-91. 